\title{
CYP19A1 mediated sex hormone metabolism promotes severe SARS-CoV-2 disease outcome in males.
}

\section{Stephanie Stanelle-Bertram}

Leibniz Institute for Experimental Virology

\section{Sebastian Beck}

Leibniz Institute for Experimental Virology

Nancy Mounogou Kouassi

Leibniz Institute for Experimental Virology

\section{Berfin Schaumburg}

Leibniz Institute for Experimental Virology

\section{Fabian Stoll}

Leibniz Institute for Experimental Virology

\section{Tian Bai}

Leibniz Institute for Experimental Virology

\section{Martin Zickler}

Leibniz Institute for Experimental Virology

\section{Georg Beythien}

Universtity of Veterinary Medicine Hannover

\section{Kathrin Becker}

Universtity of Veterinary Medicine Hannover

Madeleine de la Roi

Universtity of Veterinary Medicine Hannover

\section{Fabian Heinrich}

University Medical Center Hamburg-Eppendorf

Claudia Schulz

Universtity of Veterinary Medicine Hannover

\section{Martina Sauter}

University Hospital Tuebingen

\section{Susanne Krasemann}

University Medical Center Hamburg-Eppendorf

\section{Philine Lange}

University Medical Center Hamburg-Eppendorf 
University Medical Center Hamburg-Eppendorf

Debby van Riel

Erasmus Medical Center

Lonneke Leijten

Erasmus Medical Center

Lisa Bauer

Erasmus Medical Center

Thierry P.P. van den Bosch

Erasmus Medical Center

Boaz Lopuhaä

Erasmus Medical Center

Tobias Busche

University Bielefeld

Daniel Wibberg

University Bielefeld

Dirk Schaudien

Fraunhofer Institute for Toxicology and Experimental Medicine ITEM

Torsten Goldmann

Research Center Borstel

Hanna Jania

Leibniz Institute for Experimental Virology

\section{Zacharias Müller}

Leibniz Institute for Experimental Virology

Vinicius Pinho dos Reis

Leibniz Institute for Experimental Virology

Vanessa Krump-Buzumkic

Universtity of Veterinary Medicine Hannover

Martin Wolff

Research Center Borstel

Chiara Fallerini

University of Siena

Elisa Frullanti

University of Siena

Katrina Norwood

Helmholtz Center for Infection Research

Maren von Köckritz-Blickwede

University of Veterinary Medicine Hannover

Maria Schroeder

University Medical Center Hamburg-Eppendorf 


\section{Dominik Jarczak}

University Medical Center Hamburg-Eppendorf

\section{Axel Nierhaus}

University Medical Center Hamburg-Eppendorf

\section{Tobias Welte}

Hannover Medical School

\section{Stefan Kluge}

University Medical Center Hamburg-Eppendorf

\section{Alice C. McHardy}

Helmholtz Center for Infection Research

\section{GEN-COVID Multicenter Study}

Alessandra Renieri

University of Siena

\section{Frank Sommer}

University Medical Center Hamburg-Eppendorf

\section{Jörn Kalinowski}

University Bielefeld

\section{Susanne Krauss-Etschmann}

Research Center Borstel

Jan von der Thüsen

Erasmus Medical Center

\section{Benjamin Ondruschka}

University Medical Center Hamburg-Eppendorf

\section{Wolfgang Baumgärtner}

University of Veterinary Medicine Hannover https://orcid.org/0000-0001-8151-5644

\section{Karin Klingel}

University Hospital Tuebingen

Gabriel Guelsah ( $\nabla$ guelsah.gabriel@leibniz-hpi.de)

Leibniz Institute for Experimental Virology

\section{Research Article}

Keywords: CYP19A1, COVID-19, Sex disparity, Males

Posted Date: December 8th, 2021

DOl: https://doi.org/10.21203/rs.3.rs-107474/v2

License: (c) (i) This work is licensed under a Creative Commons Attribution 4.0 International License. Read Full License 



\section{CYP19A1 mediated sex hormone metabolism promotes severe SARS-CoV-2 disease outcome in males.}

${ }^{1}$ Department for Viral Zoonoses-One Health, Leibniz Institute for Experimental Virology, Hamburg, Germany; ${ }^{2}$ Institute for Virology, University for Veterinary Medicine Hannover, Hannover, Germany; ${ }^{3}$ German Center for Infection Research (DZIF), ${ }^{4}$ Department of Pathology, University of Veterinary Medicine Hannover, Hannover, Germany; ${ }^{5}$ Institute of Legal Medicine, University Medical Center Hamburg-Eppendorf, Germany; ${ }^{6}$ Research Center for Emerging Infections and Zoonoses, University of Veterinary Medicine Hannover, Hannover, Germany; ${ }^{7}$ Institute for Pathology and Neuropathology, University Hospital Tuebingen, Germany; ${ }^{8}$ Institute of Neuropathology, University Medical Center Hamburg-Eppendorf, Germany; ${ }^{9}$ Core Facility Experimental Pathology, University Medical Center Hamburg-Eppendorf, Hamburg, Germany;

${ }^{10}$ Department of Viroscience, Erasmus Medical Center, Rotterdam, The Netherlands; ${ }^{11}$ Department of Pathology, Erasmus University Medical Center Rotterdam, The Netherlands; ${ }^{12}$ Microbial Genomics and Biotechnology, Center for Biotechnology (CeBiTec), University Bielefeld, Germany; ${ }^{13}$ Fraunhofer Institute for Toxicology and Experimental Medicine ITEM,

Hannover, Germany; ${ }^{14}$ Pathology of the University Medical Center Schleswig-Holstein, Campus Lübeck and the Research Center Borstel, Research Center Borstel, Leibniz Center for Medicine and Biosciences, Borstel, Germany; ${ }^{15}$ Early Life Origins of Chronic Lung Disease, Research Center Borstel, Leibniz Lung Center, Member of the German Center for Lung Research (DZL), Borstel, Germany; ${ }^{16} \mathrm{Med}$ Biotech Hub and Competence Center, Department of Medical Biotechnologies, University of Siena, Italy; ${ }^{17}$ Medical Genetics, University of Siena, Italy; ${ }^{18}$ Department for Computational Biology of Infection Research, Helmholtz Center for Infection Research, Braunschweig, Germany; ${ }^{19}$ Braunschweig Integrated Centre of Systems Biology (BRICS),Technische Universität Braunschweig, Braunschweig, Germany; ${ }^{20}$ Department of Biochemistry,

University of Veterinary Medicine Hannover, Hannover, Germany; ${ }^{21}$ Department of Intensive Care Medicine, University Medical Center Hamburg-Eppendorf, Hamburg, Germany; ${ }^{22}$ Department of Respiratory Medicine, Hannover Medical School, Hannover, Germany; ${ }^{23}$ Biomedical Research in Endstage and Obstructive Lung Disease, Member of the German Center for Lung Research, Hannover, Germany; ${ }^{24}$ Cluster of Excellence RESIST (EXC 2355), Hannover Medical School, Hannover, Germany; ${ }^{25}$ Genetica Medica, Azienda Ospedaliero-Universitaria Senese, Italy; ${ }^{26}$ Division Men's Health, University Medical Center Hamburg-Eppendorf, Hamburg, Germany; ${ }^{27}$ Institute for Experimental Medicine, ChristianAlbrechts-Universität zu Kiel, Germany; 
Male sex belongs to one of the major risk factors for severe COVID-19 outcome. However, underlying mechanisms that could affect sex dependent disease outcome are yet unknown. Here, we identified the CYP19A1 gene encoding for the testosterone-to-estradiol metabolizing enzyme CYP19A1 (alias aromatase) as a male abundant host factor that contributes to worsened disease outcome in SARS-CoV-2 infected male hamsters. Pulmonary CYP19A1 transcription is further elevated upon viral infection in males correlating with reduced testosterone and increased estradiol levels. Dysregulated circulating sex hormone levels in male golden hamsters are associated with reduced lung function compared to females. Treatment of SARS-CoV-2 infected hamsters with letrozole, a clinically approved CYP19A1 inhibitor, supported recovery of dysregulated plasma sex hormone levels and was associated with improved lung function and health in male but not female animals compared to placebo controls. Whole human exome sequencing data analysis using a Machine Learning approach revealed a CYP19A1 activity increasing mutation being associated with the development of severe COVID-19 for men. In human autopsy-derived lungs CYP19A1 was expressed to higher levels in men who died of COVID-19, at a time point when most viral RNA was cleared. Our findings highlight the role of the lung as a yet unrecognized but critical organ regulating metabolic responses upon respiratory virus infection. Furthermore, inhibition of CYP19A1 by the clinically approved 66 drug letrozole may pose a new therapeutic strategy to reduce poor long-term COVID-19 outcome. 


\section{Introduction}

The COVID-19 pandemic continues to threaten the human population since its first emergence in China. To date, $\sim 241$ million people have been infected and $\sim 5$ million people have died worldwide since pandemic declaration on $11^{\text {th }}$ March 2020 (as of November 2021) ${ }^{1}$. Retrospective analysis of epidemiologic data revealed that men, older age and underlying metabolic diseases, such as obesity, diabetes type II and arterial hypertension pose high risks for fatal COVID-19 ${ }^{2-6}$. Increased COVID-19 case fatality rate in males is not reflected by increased SARS-CoV-2 male incidence compared to females ${ }^{3,4,7}$. Thus, it is unlikely that male prevalence in COVID-19 death can be explained by elevated susceptibility to viral infection.

Factors that mediate sex disparity in COVID-19 outcome might include a complex interaction of biological sex differences (e.g. chromosomes, sex steroids), gender aspects (e.g. social behavior) and underlying metabolic disorders ${ }^{6,8}$. Sex differences of genetic origin are constant throughout life, while sex differences originating from hormonal effects may alter with increasing age or in dependency of metabolic health as observed in COVID-19 patients. It is known that testosterone levels in men and estradiol levels in women are reduced with increasing age in general ${ }^{9}$. Several independent studies highlighted that sex hormone levels are dysregulated in males hospitalized with COVID-19 in contrast to females with COVID-19 ${ }^{10-}$ 13. Thus, there is increasing evidence that the sex hormone metabolism might play a crucial role in COVID-19 sex disparities, albeit underlying mechanisms remain elusive.

Sex hormones act as master regulators of host cell immunity and exert functions far beyond reproduction. Intracellular androgen and estrogen receptors act as transcription factors by binding to hormone response elements in the promoter sites of many genes thereby regulating cell responses to various environmental changes. Cell surface bound androgen and estrogen receptors may additionally affect host responses. Both, intracellular and cell surface mediated sex hormone receptors may upon activation affect cell-to-cell signaling (paracrine effects) or systemic hormone signaling pathways via the blood system (endocrine effects) ${ }^{14}$.

In the last decades, evidence grew that sex hormones play a pivotal role in lung health. Sex differences are known in various lung diseases, such as asthma, COPD and pulmonary fibrosis ${ }^{15}$. Research in preterm neonates, where respiratory distress is one of the major causes of death, highlighted a protective role of androgens in lung development ${ }^{16}$. Conversely, clinical and 
experimental studies identified an inflammatory function for estrogens and their metabolites, 103 such as estradiol ${ }^{5,17}$. Physiological changes in sex hormone levels, such as those occurring 104 during the menstrual cycle, pregnancy and menopause were associated with changes in lung 105 function ${ }^{18,19}$.

107 In this study, we sought to study the underlying mechanisms involved in sex dependent SARS-

$108 \mathrm{CoV}-2$ pathogenesis using the golden hamster model. Combining endocrine, immune and lung 109 assessments, we identified the testosterone-to-estradiol converting enzyme CYP19A1 as a 110 factor that contributes to worsened disease outcome in males. Treatment with the clinically 111 approved drug letrozole recovered dysregulated sex hormone levels upon SARS-CoV-2 112 infection resulting in improved lung health in males but not females. Whole-lung transcriptome 113 analysis identified CYP19A1 regulated pathways that are associated with improved lung health 114 in males. Human whole exome sequencing analysis by machine learning using data obtained 115 from 2,866 COVID-19 patients identified a CYP19A1 activity increasing mutation associated 116 with severe disease outcome in men but not women. In line, human autopsy-derived lung analysis revealed an elevated expression of CYP19A1 in men deceased with COVID-19 unlike women or non-COVID-19 controls. 
SARS-CoV-2 infection causes severe and prolonged respiratory dysfunction in male hamsters.

In order to assess whether SARS-CoV-2 infection causes sex differences in pathogenesis, we infected male and female hamsters with the same virus dose using the moderate COVID-19 model in golden hamsters ${ }^{20,21}$. During the first six days of acute infection, weight loss was comparable between males and females (Figure S1A). However, during the recovery phase from day 7 until day 14 post infection (p.i.), infected males recovered more slowly compared to females. While infected females regained their initial weight on day14 p.i., infected males still showed reduced body weight (Figure S1A). The overall pathogenesis was also reflected in the lung, where SARS-CoV-2 infected males and females showed similar lung pathologies (e.g. inflammation, alveolar destruction) on day 3 p.i. (Figure S1B). However, at day 14 p.i., after complete virus clearance in males and females (data not shown) male lungs showed local fibrosis in contrast to females (Figure S1C).

Next, we longitudinally measured respiratory function in SARS-CoV-2 infected males and females on day 6 (defined as acute phase) as well as days 14 and 21 p.i. (defined as recovery phase) (Figure 1, Figure S2). Respiratory function was more severely impaired in infected males compared to females on day 6 p.i.. In both sexes, the tidal volume was reduced on day 6.p.i., which persisted in males until day 14 p.i. (Figure 1 A and B). Male hamsters compensated this deficit by a higher breathing frequency, resulting in a normal ventilation rate (Figure 1A and C, Figure S2A). In contrast, the breathing frequency stayed normal in females that showed a similar reduced tidal volume as males, at the cost of a slightly reduced ventilation rate (Figure 1B and D; Figure S2B). In line with restrictive lung disease, the expiratory flow was enhanced in male hamsters, while their inspiratory flow was reduced (Figure 1E, G and I). These changes remained visible in part until day 14 p.i. (Figure 1 and Figure S2). Again, infected female hamsters had a normal expiratory flow with a reduction in inspiratory flow only

Taken together, the results of the comprehensive lung function tests show that SARS-CoV-2 infected female hamsters recover on days 14 and 21 p.i.. In contrast, infected male hamsters (Figure 1F, $\mathbf{H}$ and $\mathbf{J})$. In- and expiratory pauses were shortened both in males and females (Figures S2C-F). still present an impaired respiratory capacity on day 14 p.i.. Thus, male hamsters undergo more 
severe and prolonged lung function deficits compared to female hamsters upon SARS-CoV-2 infection.

\section{SARS-CoV-2 infection causes low testosterone and high estradiol levels in the plasma of} male hamsters.

As pulmonary dysfunction showed sex differences, we aimed to identify potential underlying mechanisms. However, no sex differences were detected in SARS-CoV-2 replication in the lung or in cytokine and chemokine levels (Figure S3), suggesting other underlying mechanisms. Changes in sex hormones are well recognized to affect lung function in a sexdependent fashion. Thus, we wanted to assess whether SARS-CoV-2 infection interferes with sex hormone homeostasis. Therefore, we next measured levels of the key sex hormones testosterone and estradiol at various time points after viral infection.

In infected male hamsters, testosterone levels dropped on day 3 p.i. by $\sim 80 \%$ compared to levels prior to infection but started to recover on day 6 p.i. and fully recovered on day 14 p.i. compared to males treated with the immunostimulant poly(I:C) or PBS (Figure 2A). Virus replication in the male lungs was negatively associated with testosterone levels. Lowest plasma testosterone levels detected on day 3 p.i. coincided with highest virus titers in the lung of SARS-CoV-2 infected male hamsters (Figure 2B). Conversely, estradiol levels increased by $\sim 1300 \%$ in the plasma of infected male animals on day 3 p.i. compared to PBS treated groups, and remained elevated on day 6 p.i. and mostly recovered on day 14 p.i. (Figure 2C). Estradiol levels were positively associated with virus titers in the lung. Highest estradiol levels in the plasma were associated with peak virus titers detected in the lung of infected male hamsters (Figure 2D).

In infected female hamsters, no significant alteration in plasma testosterone levels was detected in line with the very low levels of testosterone in young female hamsters in general (Figure 2E). High virus titers were detected on day 3 p.i. in females, comparable to the virus dynamics detected in males (Figure 2F). Estradiol levels, however, were reduced by $\sim 80 \%$ in SARSCoV-2 infected female hamsters compared to the respective PBS control group (Figure 2G). Virus titers were negatively associated with estradiol levels in infected female hamsters on day 3 p.i. (Figure $\mathbf{2 H}$ ). 
These findings show that SARS-CoV-2 infection causes dysregulation in circulating sex hormones. In males, viral infection is associated with low testosterone and high estradiol levels. In females, viral infection is associated with low estradiol levels. In both, sex hormone levels are almost fully recovered at day 14 p.i. when no replicating virus is detectable anymore.

\section{CYP19A1 transcription is highly induced upon SARS-CoV-2 infection in the lung of male} hamsters.

The conversion of testosterone-to-estradiol by aromatization occurs via the CYP19A1 gene (alias aromatase), which is localized in the endoplasmic reticulum and is expressed in a wide range of tissues, including epithelial cells, endothelial cells, the vascular wall and macrophages in various mammalian species ${ }^{22-24}$. The CYP19A1 gene contains predicted binding sites for key transcription factors, such as for cytokine induced NF-kB, STAT1,-3,-4,-6 and many others (Figure 3A). In addition, the promoter region of CYP19A1 contains binding sites for sex hormone responsive elements, such as binding sites for the androgen receptor (AR), estrogen receptor $(\mathrm{ER}-\alpha / \beta)$, progesterone receptor $(\mathrm{PR}-\alpha / \beta)$ as well as SF-1 (steroidogenic factor-1; involved in sex determination by controlling activity of genes related to the reproductive glands or gonads and adrenal glands) ${ }^{25-29}$ indicating a critical role for CYP19A1 in sex-specific immunity (Figure 3A). Thus, we assessed whether SARS-CoV-2 infection may affect CYP19A1 transcription in the lung.

However, first, we compared CYP19A1 protein expression in male versus female lungs. We detected increased CYP19A1 expression in the male lung compared to the female lung (Figure 3B, Figure S4). This is in line with previous reports that CYP19A1 is expressed more abundantly in males compared to females ${ }^{30}$. Upon SARS-CoV-2 infection, in male hamsters, CYP19A1 transcription was increased up to $\sim 20$ times compared to poly(I:C) or PBS treated controls at day 3 p.i. (Figure 3C), then recovering slowly on day 6 p.i. and fully on day 14 p.i. (Figure 3D and E). Among 13 different cytokines and chemokines assessed, MIP-1 $\alpha$, MIP-1ß, Eotaxin and VEGF showed the strongest dysregulation in the male lung. MIP-1 $\alpha$, MIP-1ß and Eotaxin levels were strongly induced in the lungs of SARS-CoV-2 infected male hamsters, while VEGF levels were suppressed at day 3 p.i. (Figure S3). Interestingly, the elevated levels of MIP-1 $\alpha$, MIP-1 $\beta$ and Eotaxin and the reduced level of VEGF remained in the lungs of virus infected male animals on day 6 p.i., a time point where most animals cleared virus infection (Figure S3). Regression analysis revealed a positive association for MIP-1 $\alpha$ and a negative 
association for VEGF with CYP19A1 mRNA expression (Figure 3F-I). Regression analysis for the sex hormones revealed a negative association of testosterone and a positive association of estradiol with CYP19A1 transcription in SARS-CoV-2 infected male hamsters (Figure 3J and K). This is likely the consequence from CYP19A1 mediated conversion of testosterone-toestradiol further supporting the role of pulmonary CYP19A1 in sex hormone metabolism in virus infected males.

In the lungs of SARS-CoV-2 infected female hamsters, CYP19A1 transcription was increased up to 10-times compared to PBS or poly(I:C) treated control females on day 3 p.i., recovered on day 6 p.i. and was even decreased on day 14 p.i. (Figure S5A-C). Similar to SARS-CoV-2 infected males, the most significantly dysregulated chemokines were MIP- $1 \alpha$, MIP-1ß, Eotaxin and VEGF. On day 3 and 6 p.i., MIP-1 $\alpha$, MIP-1ß and Eotaxin levels were significantly elevated in contrast to VEGF levels that were significantly reduced in infected female hamsters as compared to uninfected control groups (Figure S3). Regression analysis revealed a positive association for MIP-1 $\alpha$ and Eotaxin with CYP19A1 mRNA expression (Figure S5D-G). Regression analysis for the sex hormones did not reveal any significant association with CYP19A1 transcription in SARS-CoV-2 infected female hamsters (Figure S5H and I).

In order to assess whether SARS-CoV-2 infection additionally affects the transcription of other sex hormone metabolizing enzymes in the lung, we measured mRNA levels of $5 \alpha$-reductase and 17ß-hydroxysteroid dehydrogenase. None of these steroid metabolizing enzymes were transcriptionally dysregulated in the lungs of SARS-CoV-2 infected male or female hamsters (Figure S6) further supporting a specific role for CYP19A1 in SARS-CoV-2 infection in the lung. Remarkably, CYP19A1 mRNA or protein levels were also not affected in other organs known to express CYP19A1 abundantly, such as the gonads and the brain despite detection of SARS-CoV-2 replication (Figure $\mathbf{S 7}$ and S8).

These data suggest that SARS-CoV-2 infection mediates specific elevation of CYP19A1 transcription in the lung, most pronounced in male animals. Regression analyses suggest a positive regulatory role for MIP-1 $\alpha$ on the transcriptional regulation of CYP19A1 in infected male and female hamsters. In contrast, sex-dependent regulation of CYP19A1 transcription is detected for VEGF in males and for Eotaxin in females. Moreover, the negative association of circulating testosterone and the positive association of circulating estradiol levels in SARS- 
CoV-2 infected males unlike females further supports the concept that pulmonary CYP19A1 plays a crucial role in sex hormone metabolism in males.

\section{SARS-CoV-2 unlike SARS-CoV or H1N1 influenza induces CYP19A1 transcription in} in vitro lung cultures.

We next sought to understand whether CYP19A1 mRNA dysregulation in the lung is dependent on the complex in vivo setting or whether potential alterations could also be recapitulated in vitro. Therefore, we infected human lung cells (Calu-3) with SARS-CoV-2 or as controls, with SARS-CoV or H1N1 influenza A virus. CYP19A1 mRNA induction was detected up to 40 times in SARS-CoV-2 infected but not in SARS-CoV or H1N1 influenza virus infected lung cells (Figure 3L).

This finding additionally supports the role of respiratory tissues in sex hormone metabolism. It further indicates that SARS-CoV-2 has evolved specific mechanisms not observed in its ancestor or an unrelated respiratory pathogen to dysregulate a major sex hormone metabolizing enzyme specifically in the lung.

Estrogen receptor (ESR)-B expression is increased in the lung of SARS-CoV-2 infected male hamsters.

We next assessed whether SARS-CoV-2 infection might alter sex hormone receptor expression that could additionally affect paracrine signaling in the lung. In SARS-CoV-2 infected hamsters, we detected dysregulated expression of estrogen receptors (ESR- $\alpha$, ESR- $\beta$ ) but not androgen receptors (AR, ZIP9) (Figure 3M-P, Figure S5J-M). In males and females, viral infection caused reduced expression of the ESR- $\alpha$ receptor as compared to poly(I:C) or PBS treated controls (Figure 3M, Figure S5J). In males unlike females, SARS-CoV-2 infection additionally caused increased expression of ESR-ß (Figure 3N, Figure S5K).

These data suggest that dysregulated expression of ESR- $\beta$ in the lung may present a paracrine mode-of-action for estradiol contributing to local inflammation in males. 

inhibitor letrozole improves lung health in males.

The data obtained so far suggest that dysregulated circulating sex hormones as well as altered expression of pulmonary sex hormone receptors might affect endocrine and paracrine signaling affecting overall pathogenesis and recovery. In order to determine the role of CYP19A1 in these alterations, we treated SARS-CoV-2 infected male and female hamsters with the clinically approved CYP19A1 inhibitor letrozole (Figure 9A). Letrozole is used in humans for the treatment of estrogen receptor positive breast cancer in women ${ }^{31}$ as well as to treat hypogonadism in men ${ }^{32}$. Letrozole treatment resulted in elevated testosterone levels in virus infected males, albeit statistically not significant (Figure 9B). Estradiol levels could be significantly reduced upon letrozole treatment in infected males (Figure 9C). In females, letrozole treatment led to significantly increased testosterone levels and a tendency towards reduced estradiol levels (Figure 9D and E). Thus, in males and females, letrozole treatment shifted the testosterone-to-estradiol ratio suggestive of successful CYP19A1 functional inhibition in both sexes as described before ${ }^{31,32}$.

Letrozole treatment also affected sex hormone receptor expression. ESR- $\alpha$ transcription levels were significantly reduced in SARS-CoV-2 infected male hamsters upon letrozole treatment (Figure 9H) in line with reduced plasma estradiol levels (Figure 9C). Albeit statistically not significant, there was also a trend towards elevated AR transcription in letrozole treated males (Figure 9F) in line with elevated plasma testosterone levels (Figure 9B). In SARS-CoV-2 infected females, letrozole treatment did not affect androgen or estrogen receptor expression (Figure 9J-M). Letrozole treatment did not affect virus titers in the lung or cytokine/chemokine responses in males and females compared to placebo treated groups (Figure S10).

We then assessed whether recovered sex hormone levels might result in improved lung function in SARS-CoV-2 infected males and females. In PBS male and female controls, treatment with letrozole or placebo did not affect lung function ruling out adverse effect in this regard (Figure S11, S12). In males infected with SARS-CoV-2, letrozole treatment resulted in faster recovery of the tidal volume from day 6 p.i. onward ultimately attaining the level of the placebo group. Accordingly, the breathing frequency showed accelerated normalization in letrozole treated animals, resulting in a longer inspiratory time (Figure 4A-D, Figure S13). This correlated histologically with less collapsed alveoli, less immune cell infiltrates in adjacent alveolar spaces and less alveolar wall thickening in the lungs of SARS-CoV-2 infected males upon letrozole 
treatment (Figure 4E). In line, letrozole treated males presented less fibrotic structures on day 14 p.i. compared to placebo treated males (Figure 4F). Overall, these findings were reflected by an increased weight gain of letrozole treated males compared to placebo males on day 21 p.i. (Figure 4G). In contrast, letrozole treatment did not improve lung function in SARS-CoV2 infected females at any time point (Figure 4H-K). In line, neither lung pathology nor weight was not significantly improved in letrozole treated infected females as compared to placebo controls (Figure 4L-N).

These findings confirm that CYP19A1 plays an important role in SARS-CoV-2 pathogenesis in males. Treatment with the clinically approved CYP19A1 inhibitor letrozole substantially improves male lung health.

\section{Whole-lung transcriptome analysis identifies letrozole treatment modified pathways that} are associated with improved lung health in SARS-CoV-2 infected male hamsters.

To investigate which pathways might be modulated in SARS-CoV-2 infected male lungs that impair full recovery and how letrozole treatment contributes to improved lung health, we performed whole-lung transcriptome profiles on day 21 p.i.. Therefore, we compared male and female hamsters infected with SARS-CoV-2 and treated either with letrozole or placebo. As negative controls, we included males and females inoculated with PBS and then treated with letrozole or placebo. A total of 120 million sequence reads (on average approx. 4 million reads for each of five replicates per condition) amounting to $103 \mathrm{~Gb}$ sequence information were generated for all transcriptome libraries. Quality filtering resulted in high quality sequence reads that were mapped onto the Syrian hamster draft genome sequence. For all conditions, the amount of mapped hamster reads was about $92 \%$ of all reads. We found that differential genes were expressed in males and females infected with SARS-CoV-2 (Figure 5A and B). Interestingly, the transcriptomic profile significantly changed in the infected male lungs treated with letrozole (Figure 5C) in contrast to letrozole treated infected females (Figure 5D). In SARS-CoV-2 infected males and females a total of 256 genes were up-regulated of which 188 were male- and 57 female-specific and another 11 shared by both sexes (Figure 5A-E). A total of 56 genes were down-regulated in SARS-CoV-2 infected animals with 6 being male-specific, 49 female-specific and 1 common gene. Letrozole treatment resulted in the down-regulation of 133 von 199 up-regulated genes in infected males (Figure 5F). In contrast, in letrozole treated infected females, 65 out of 68 genes remained unaltered (Figure 5G). Thus, letrozole treatment 
results in sex-specific alterations in the transcriptomic profile of the lung. Herein, improved lung health in SARS-CoV-2 infected male hamsters upon letrozole treatment was associated with changes in many pathways of which those involved in lipid and protein processes, e.g. lipid oxidation (GO:0034440), lipid modification (GO:0030258) and protein phosphorylation (GO:0006468) were among the most significant (Figure 5H).

These data show that letrozole treatment can recover most of the dysregulated genes in the lungs of SARS-CoV-2 infected males but not females. Furthermore, the recovery of these dysregulations by the CYP19A1 inhibitor letrozole confirms the pivotal role of CYP19A1 in SARS-CoV-2 pathogenesis in males.

\section{CYP19A1 activity increasing mutation Thr201Met is associated with the development of} severe COVID-19 in men.

We next sought to translate our findings into humans. Mutations in the CYP19A1 gene were described before to affect disease outcome and treatment efficacies with CYP19A1 inhibitors in a sex-specific manner ${ }^{33,34}$. Thus, we analyzed human exome data obtained from 2,866 COVID-19 patients (GEN-COVID; https://sites.google.com/dbm.unisi.it/gen-covid) ${ }^{35,36}$ and applied the post-Mendelian model using a Machine Learning approach in combination with a Boolean representation of whole exome sequencing (WES) data as described previously ${ }^{37,38}$ (Figure 6). Demographic and clinical characteristics of the GEN-COVID cohort were described previously ${ }^{35,36}$ (Figure 6A-E). The model identified the coding low-frequency Thr201Met variant of CYP19A1 as being associated to COVID-19 severity in males, but not in females. The association between Thr201Met and severity is remarkable with an OR 1.96 in males (95\% CI, 1.2 to 3.16; p-value 0.006) (Figure 6F and G).

These data suggest an association of the Thr201Met mutation in CYP19A1, which was described before to increase CYP19A1 enzyme activity ${ }^{39}$, with severe COVID-19 in men but not women. 


\section{CYP19A1 is highly expressed in autopsy-derived lung tissues of men who died of COVID-} 19.

Next, we sought to assess whether an increased CYP19A1 expression could also be detected in patients who died of COVID-19 as a potentially contributing factor. Therefore, we analyzed autopsy material from the lungs of men and women who were positive for SARS-CoV-2 by qRT-PCR and were diagnosed later to have died of COVID-19 ( $n=57)$ at three independent study sites (Tübingen ( $n=8$ males, $n=3$ females), Hamburg ( $n=26$ males, $n=8$ females) and Rotterdam ( $n=12$ males, no female cases)). As controls, we analyzed lung material obtained from men and women who were never diagnosed positive for SARS-CoV-2 by qRT-PCR and who were diagnosed to have died for other reasons (herein referred as non-COVID-19 control group) (Figure 7).

At all three study sites, CYP19A1 was abundantly expressed in the lungs of COVID-19 males compared to non-COVID-19 male controls (Figure 7). CYP19A1 was also expressed in some lungs of deceased COVID-19 females compared to non-COVID-19 female controls, albeit to a lesser extent than in males (Figure 7A and B). However, due to the overall lower case of female COVID-19 deaths compared to males, conclusive interpretation in females is limited.

In general, CYP19A was expressed in epithelial cells, in endothelial cells but most profoundly in macrophages at all three independent study sites. Noteworthy, SARS-CoV-2 NP protein or RNA was still detectable in the lungs of most deceased females, while viral antigen or RNA was cleared at the time point of death in most males (Figure 7A and B). Consecutive staining of the lung autopsies revealed still highly CYP19A1 expressing macrophages in COVID-19 males, albeit SARS-CoV-2 RNA was no longer detectable at the time point of death (Figure 7A-C). On the other hand, consecutive staining in COVID-19 females shows that SARS-CoV2 RNA positive macrophages overlay with CYP19A1 expressing macrophages (Figure 7A). This findings suggests a potential role of macrophages in virus replication and CYP19A1 expression. Quantification of CYP19A1 mRNA levels revealed a transcriptional increase up to $\sim 50$ times in the lungs of deceased COVID-19 males in the Tübingen cohort, up to 10 times in the male COVID-19 Hamburg cohort and up to 20 times in the male COVID-19 Rotterdam cohort (Figure 7D-F). 
419 These findings show that CYP19A1 is also abundantly expressed on the mRNA and protein

420 level in the lungs of men who died of COVID-19. In deceased men, CYP19A1 expression is 421 still hauling even when virus is no longer detectable. 


\section{Discussion}

We herein identified the CYP19A1 gene as key factor contributing to increased disease pathogenesis in males infected with SARS-CoV-2 compared to females. Treatment of infected hamsters with letrozole, a clinically approved CYP19A1 inhibitor, improved lung health and overall recovery from the disease in males. In contrast, females did not substantially benefit from letrozole treatment further highlighting the role of CYP19A1 in male SARS-CoV-2 pathogenesis.

In male hamsters, we found that respiratory SARS-CoV-2 infection impaired lung function compared to infected females. Moreover, SARS-CoV-2 infection in males reduced plasma testosterone and elevated plasma estradiol levels. This finding is in agreement with several studies reporting low testosterone and high estradiol levels in the plasma of hospitalized male COVID-19 patients ${ }^{11-13}$. Follow-up studies report that even seven months after SARS-CoV-2 infection still half of the COVID-19 patients have testosterone levels below clinical references ${ }^{40}$. In the hamster model, we could show that the testosterone-to-estradiol metabolizing gene CYP19A1 was strongly induced on the mRNA and protein level in the lungs of SARS-CoV-2 infected male hamsters. CYP19A1 transcription in the infected male lung is positively associated with the MIP-1 $\alpha$ and negatively associated with VEGF. Although further studies are required to identify the transcription factors that directly regulate $C Y P 19 A 1$, our findings are in line with the presence of binding sites for transcription factors that are induced by various cytokines and chemokines ${ }^{25-29}$. Additionally, in males, CYP19A1 transcription in the infected lung was negatively associated with testosterone levels and positively associated with estradiol levels. This finding further supports the concept that pulmonary CYP19A1 converts testosterone-to-estradiol consequently resulting in changes in circulating sex hormone levels in males but not females. However, an additional regulating feedback rule by sex hormones is likely given that the CYP19A1 promoter region also contains androgen and estrogen receptor binding motives ${ }^{25-29}$.

In female hamsters, we found that respiratory SARS-CoV-2 infection also causes reduced lung function albeit clearly less severe than in infected males. SARS-CoV-2 infection caused reduced plasma estradiol levels in females. Analysis of sex hormone levels in critically ill women with COVID-19 revealed elevated rather reduced plasma estradiol levels that correlated with systemic inflammation ${ }^{13}$. However, it should be considered that most critically ill COVID19 women in the studied cohort were post-menopausal ${ }^{13}$ in contrast to the young female 
animals used in this study. Menopause was associated with accelerated decline in lung function in women ${ }^{19}$. In the context of our current data, this might explain why post-menopausal women are at higher risk than young women for COVID-19 related ICU admission. Moreover, critically ill post-menopausal COVID-19 females presented elevated testosterone levels ${ }^{13}$, which -at least theoretically- could act as a substrate for CYP19A1, thereby additionally fueling inflammatory processes in the lung. In hamsters, SARS-CoV-2 infection increased the transcription of CYP19A1 in the female lung. However, the expected elevation of estradiol levels was not observed. One reason for this might be the limited availability of the CYP19A1 substrate testosterone in young animals of reproductive age. Future investigations are needed to understand the role of sex hormones on COVID-19 outcome in pre- and post-menopausal women. However, CYP19A1 transcription in the infected female lung was positively associated with MIP-1 $\alpha$ and Eotaxine. In contrast to infected male animals, however, sex hormone expression levels were not associated with CYP19A1 transcription in the lungs of SARS-CoV2 infected female animals. This suggests that pulmonary CYP19A1 is not involved in metabolic processes in females that would affect circulating sex hormone levels.

Treatment of SARS-CoV-2 infected male and female hamsters with letrozole, a clinically approved CYP19A1 inhibitor increased circulating testosterone levels and reduced circulating estradiol levels suggestive of functional CYP19A1 inhibition in both sexes. In males, full recovery of testosterone levels (in contrast to estradiol levels) could not be achieved. One possible reason could be the presence of SARS-CoV-2 RNA in the testis of infected males that could have impaired testosterone synthesis without the involvement of CYP19A1. However, reducing circulating estradiol levels in SARS-CoV-2 infected males by letrozole treatment improved lung health with overall faster recovery in males. Thus, letrozole treated males showed improved lung function that was reflected by ameliorated lung pathology and accelerated weight gain after virus clearance. In contrast, letrozole treatment did not show any benefit for SARS-CoV-2 infected females. This finding highlights a key role of CYP19A1 in male pathogenesis upon SARS-CoV-2 infection.

Whole-lung transcriptome analysis of SARS-CoV-2 infected hamsters with letrozole or placebo treatment revealed key pathways associated with improved male outcome. In line with the failed benefit of females from letrozole treatment, there were no major differences in the lung transcriptome profiles of placebo versus letrozole treated females. In contrast, a series of gene pathways were identified in the lungs of males infected with SARS-CoV-2 upon letrozole 
treatment as compared to placebo controls. The major pathways that were differentially altered upon letrozole treatment were involved in lipid oxidation, lipid modification and protein phosphorylation. The prominent involvement of lipid pathways is interesting given that there is evidence that efficient SARS-CoV-2 replication depends on lipid metabolism ${ }^{41}$. Alveolar lipids are known to be involved in pulmonary diseases ${ }^{42}$. Similar, for both sex steroids, their involvement in wound healing were reported before ${ }^{43}$. It was shown for example that ovariectomized rats present diminished fibrosis, while estradiol replacement restored fibrotic response to that of intact females ${ }^{44}$. In mice, testosterone treatment restored asthmatic lung exacerbation by attenuating airway inflammation ${ }^{45}$. Population-based studies further supported this concept by showing strong associations between testosterone levels and lung function in men ${ }^{46}$. Although future studies are required to understand these androgen and/or estrogen controlled transcription pathways in improved long-term lung health, these data further support the concept that balanced sex hormone levels are critical for lung health.

Analyzing whole-exome sequencing data derived from 2,866 COVID-19 patients within the GEN-COVID cohort, we detected the enzyme activity increasing CYP19A1 variant Thr201Met 39 to be associated with severe COVID-19 outcome in men but not women. Letrozole was reported to inhibit the CYP19A1 Thr201Met variant as effectively as the wildtype enzyme ${ }^{39}$, suggesting that male carriers of the Thr201Met variant might still benefit from letrozole treatment. Furthermore, we detected increased expression of $C Y P 19 A 1$ in the lungs of men but not women at the time point of death- still hauling even after virus clearance.

In the human population, besides genetic variations, CYP19A1 function can be additionally modified by other conditions, such as obesity and age. CYP19A1 is highly expressed in adipose tissue where it converts testosterone-to-estradiol. The higher the amount of adipose tissue present, the higher the risk for low testosterone and elevated estradiol levels in humans, which is particularly observed in obese people ${ }^{32,47,48}$. Testosterone is produced in the gonads of boys at the age of 10-12 years, peaking in adulthood and declining slowly with increasing age. Agedependent testosterone deficiency may pose another risk factor for reduced lung health ${ }^{46}$. However, dysregulation of testosterone and/or estradiol levels is a magnitude higher in SARSCoV-2 infected men compared to age-matched healthy controls or patients with obesity. This might pose one explanation as why male patients are at high risk for severe COVID-19 outcome in particular when combined with increasing age and obesity. Interestingly, letrozole treatment was shown to normalize serum testosterone levels in obese men with hypogonadism ${ }^{32}$ 
524 suggesting that this risk group might additionally benefit from letrozole treatment upon SARS525 CoV-2 infection.

526

527 Collectively, these findings highlight that the CYP19A1 gene is involved in SARS-CoV-2 528 mediated sex disparity. Furthermore, we identified the clinically approved CYP19A1 inhibitor 529 letrozole as a potential new therapeutic strategy to improve long-term disease outcome in 530 SARS-CoV-2 infected males. 


\section{Viruses}

The SARS-CoV-2 strain (SARS-CoV-2/Germany/Hamburg/01/2020; ENA study PRJEB41216 and sample ERS5312751) was isolated by inoculation of VeroE6 cells (ATCC CCL81) with $200 \mu 1$ of a human nasopharyngeal swab of a confirmed patient with COVID-19 in Hamburg and propagated for three serial passages in VeroE6 cells. The SARS-CoV (Frankfurt 1) was grown and titrated in VeroE6 cells (kindly provided by Christian Drosten, Charité, Berlin, Germany). VeroE6 were cultivated in DMEM (Sigma-Aldrich GmbH) with 2\% fetal bovine serum (FBS), $1 \%$ penicillin-streptomycin and $1 \% \mathrm{~L}$-glutamine at $37^{\circ} \mathrm{C}$ for virus propagation. The 2009 pandemic H1N1 influenza A virus (A/Hamburg/NY1580/09; H1N1) was grown and titrated in MDCK II cells (ATCC CRL-2936). MDCK II cells were cultivated in MEM (Sigma-Aldrich $\mathrm{GmbH}$ ) with $0,2 \%$ bovine serum albumin (BSA), $1 \%$ penicillinstreptomycin and $1 \% \mathrm{~L}$-glutamine at $37^{\circ} \mathrm{C}$. All cells were tested negative for Mycoplasma sp. by PCR. All infection experiments with SARS-CoV and SARS-CoV-2 were performed in the biosafety level 3 (BSL-3) laboratory at the Heinrich Pette Institute, Leibniz Institute for Experimental Virology in Hamburg, Germany.

\section{Animal experiments}

550

551

552

553

554

555

556

557

558

559

560

561

562

563

564

Male and female golden hamsters (8-12 weeks old) were purchased from Janvier. All animals were kept under standard housing conditions $\left(21 \pm 2{ }^{\circ} \mathrm{C}, 40-50 \%\right.$ humidity, food and water ad libitum) with a 12:12 light-dark cycle at the Leibniz Institute for Experimental Virology in Hamburg, Germany. For SARS-CoV-2 infection, hamsters were anaesthetized with $150 \mathrm{mg} / \mathrm{kg}$ ketamine and $10 \mathrm{mg} \mathrm{kg}^{-1}$ xylazine by intraperitoneal injection. The animals were intranasally inoculated with $10^{5}$ plaque forming units (p.f.u.) SARS-CoV-2, mock infected with PBS or were administered with $1 \mathrm{mg} \mathrm{kg}^{-1}$ poly(I:C). Letrozole dosing was calculated for male and female hamsters by taking sex-specific half times into consideration as reported before ${ }^{49-53}$. For letrozole treatment, male and female hamsters received a final dose of $0.36 \mathrm{mg} \mathrm{kg}^{-1} \mathrm{~d}^{-1}$ or $0.18 \mathrm{mg} \mathrm{kg}^{-1} \mathrm{~d}^{-1}$ letrozole, respectively, (dissolved in DMSO, Sigma-Aldrich, L6545) diluted in sterile saline (Braun), respectively, by oral gavage (stainless-steel gavaging needles) at $3 \mathrm{~h}$ and day 1 to 3 post infection (p.i.). From day 4 to 8 p.i., hamsters received letrozole via the drinking water as water gel packages (Necta) supplemented with $1 \%$ glucose and a final concentration of $0.0018 \mathrm{mg} \mathrm{ml}^{-1}$ or $0.0036 \mathrm{mg} \mathrm{ml}^{-1}$ letrozole for female and male hamsters, respectively. As control, hamsters were treated with DMSO as vehicle. 
Body weight was monitored daily up to 14 days post infection (d p.i.). On day 1, 3, 6 and 14 p.i., five to ten animals per group were euthanized by intraperitoneal injection of an overdose of pentobarbital $(800 \mathrm{mg} / \mathrm{kg})$. Blood was drawn by cardiac puncture and collected in EDTA tubes. Blood was centrifuged for $10 \mathrm{~min}$ at $3,000 \mathrm{~g}$ and $4{ }^{\circ} \mathrm{C}$, and the plasma was stored at $80^{\circ} \mathrm{C}$. Lungs, brains and gonads were homogenized in $1 \mathrm{x}$ PBS and stored at $-80^{\circ} \mathrm{C}$. For histopathological examination, lungs, brains and gonads were fixed by immersion in $10 \%$ neutral-buffered formalin and embedded in paraffin. For total RNA isolation, lungs, brains and gonads were incubated in RNAprotect Tissue Reagent (QIAGEN), for at least 24 hours at $4{ }^{\circ} \mathrm{C}$ and stored at $-80^{\circ} \mathrm{C}$.

All animal experiments were performed in strict accordance with the guidelines of German animal protection law and were approved by the relevant German authority (Behörde für Gesundheit und Verbraucherschutz; protocols N 32/2020 and N 103/2020).

\section{Plethysmography}

Respiratory function was assessed using an unrestrained barometric whole-body plethysmography. In brief, Syrian golden hamsters infected with SARS-CoV-2 or mock infected with PBS were placed in a sealed cylindrical Buxco® WBP chamber (Rat WBP standard chamber) on day $0,6,14$ and/or 21 p.i., and changes in pressure caused by tidal breathing movements were measured using a volumetric flow transducer (FP WBP Flow Transducer w/T\&HF, Data Sciences International) and amplified. Flow fluctuations were recorded using FinePointe Software System (v2.9.0, Data Sciences International). At the beginning of each session the plethysmograph was calibrated. Several parameters were obtained, including breath frequency (breaths/min), tidal volume ( $\mathrm{Ti}, \mathrm{ml})$, expiratory flow rate at the point $50 \%$ of tidal volume is expired (EF50, $\mathrm{ml} / \mathrm{sec}$ ), peak inspiratory flow rate (PIFb, $\mathrm{ml} / \mathrm{sec})$, peak expiratory flow rate $(\mathrm{PEFb}, \mathrm{ml} / \mathrm{sec})$, minute volume $(\mathrm{ml} / \mathrm{min})$, end inspiratory pause (EIP, ms), end expiratory pause (EEP, ms), inspiratory time ( $\mathrm{Ti}, \mathrm{sec})$ and expiratory time (Te, sec). Measurements were performed for $10 \mathrm{~min}$, after $20 \mathrm{~min}$ (females) or $30 \mathrm{~min}$ (males) acclimatisation periods.

\section{Histology and immunohistochemistry in hamsters}

Tissues were routinely embedded in paraffin and evaluated via light microscopy of hematoxylin and eosin (HE) as well as Azan stained slides.

Immunohistochemical detection of SARS-CoV-2 nucleoprotein (NP), CYP19A1, cluster of differentiation (CD) 204, von-Willebrandt-factor (vWF), CD31, CD3 and smooth muscle actin 
(SMA) in the golden hamster lungs was performed using the EnVision+ System (Dako Agilent Pathology Solutions). Serial sections of tissue were dewaxed and rehydrated in isopropanol and $96 \%$ ethanol followed by blockage of endogenous peroxidase by incubation in $85 \%$ ethanol with $0.5 \% \mathrm{H}_{2} \mathrm{O}_{2}$ for $30 \mathrm{~min}$ at room temperature. Antigen retrieval was performed by incubation in citrate $(10 \mathrm{mM}$ citric acid, $0.05 \%$ Tween 20$)$ or citrate- $\mathrm{Na}_{2} \mathrm{H}_{2}$ EDTA buffer $(10 \mathrm{mM}$ citric acid, $2 \mathrm{mM} \mathrm{Na} \mathrm{H}_{2} \mathrm{EDTA}, 0.05 \%$ Tween 20) for $20 \mathrm{~min}$ in a microwave at $800 \mathrm{~W}$, followed by $20 \mathrm{~min}$ at room temperature. Sections were afterwards transferred to Shandon Coverplates ${ }^{\mathrm{TM}}$ (Thermo Electron $\mathrm{GmbH}$ ) and stained with either polyclonal antibodies directed against CYP19A1 (Abcam, ab18995, 1:500), CD31 (Acris, AP15436PU, 1:100), CD204 (Abnova Corporation, MAB1710, 1:500) and vWF (Dako Agilent Pathology Solutions, 20051017, 1:500) or monoclonal antibodies against SMA (Dako Agilent Pathology Solutions, 20071964, 1:100), CD3 (Dako Agilent Pathology Solutions, A0452, 1:100) and SARS-CoV-2 NP (Sino Biological, 40143-MM05, 1:32,000) over night at $4^{\circ} \mathrm{C}$. Antibodies were diluted in 1x PBS containing $1 \%$ BSA and addition of 0,3\% Triton X-100 for the CYP19A1, SMA and SARSCoV-2 NP stainings. Sections were subsequently rinsed, and the peroxidase-labeled polymer was applied as secondary antibody for $30 \mathrm{~min}$. Visualization of the reaction was accomplished by incubation in chromogen 3,3-diaminobenzidine tetrahydrochloride (DAB, 0.05\%) and $0.03 \% \mathrm{H}_{2} \mathrm{O}_{2}$ in $1 \mathrm{x}$ PBS for $5 \mathrm{~min}$ and afterwards counterstained with Mayer's hematoxylin for 1 min. For negative controls, the primary antibody was replaced by either rabbit normal serum $(1: 3,000)$ or ascites fluid from Balb/c mice $(1: 1,000)$. The serial stainings were afterwards analyzed by light microscopy.

For the detection of collagen, picro sirius red stain kit (PSR-1-IFU, Sky tekLogan, Utah, USA) was used according to the instructions of the manufacturer.

For the quantification of Azan positive collagen fiber content in the lungs and CYP19A1 expressing tissue in lungs and gonads, slides were digitized using the Hamamatsu NanoZoomer S210 (Hamamatsu Photonics, Herrsching am Ammersee. Germany) slide scanner. Image analysis was performed on the whole-slide images. Initially lung tissue was detected using a specifically trained Bayesian classifier followed by DAB positive and negative (tissue) pixel count for CYP19A1 staining or Azan positive and negative pixel count for collagen detection using the Visiopharm Software (Visiopharm, Hørsholm, Denmark). Percentage of CYP19A1 positive tissue and Azan positive collagen fiber content was calculated for each lung section present on a slide individually and a median expression value was calculated for each animal. 


\section{Histology and immunohistochemistry in fatal COVID-19 cases}

For immunohistochemical detection of CYP19A1 in the lungs of deceased COVID-19 patients tissue probes were fixed in $4 \%$ phosphate-buffered formaldehyde and embedded in paraffin. For immunohistochemistry at the University Hospital Hamburg-Eppendorf, sections were cut at $2 \mu \mathrm{m}$. After dewaxing and inactivation of endogenous peroxidases (PBS/3\% hydrogen peroxide), antibody specific antigen retrieval was performed using the Ventana Benchmark XT machine (Ventana, Tuscon, Arizona, USA). Sections were incubated with anti-CYP19A1 (Abcam, ab18995, 1:400) or anti-SARS-CoV-2 nucleoprotein antibody (Synaptic Systems, \#HS-452 011; 1:2,000; Göttingen, Germany). For detection of specific binding, the Ultra View Universal DAB Detection Kit (Ventana, Roche) was used, which contains secondary antibodies, DAB stain and counter staining reagent for detection of nuclei. Staining were evaluated by an experience pathologist (SK) and representative images were taken with a Leica DMD108 digital microscope.

For immunohistochemistry at the University Hospital Tübingen, immunohistochemical analysis was performed by using the polyclonal antibody directed against CYP19A1 (Abcam, ab18995, 1:400) on an automated immunostainer following the manufacturer's protocol (Benchmark; Ventana Medical Systems, Tucson, AZ) and using the ultraView detection system (Ventana) and diaminobenzidine as substrate. Tissue sections were counterstained with hematoxylin. For all deceased patients, informed consent had been obtained from the next of kin $(097 / 2021 \mathrm{BO} 2)^{54}$.

For immunohistochemistry at the Erasmus Medical Center Rotterdam, immunohistochemistry was performed with an automated, validated and accredited staining system (Ventana, Tuscon, Arizona, USA) using optiview universal DAB detection Kit. In brief, following deparaffinization and heat-induced antigen retrieval for $32 \mathrm{~min}$ with $\mathrm{CC} 1$ the tissue samples were incubated with rabbit-anti-human CYP19A1 (Abcam, ab18995, 1:400) for 32 minutes. Incubation was followed by hematoxylin II counter stain for $12 \mathrm{~min}$ and then a blue colouring reagent for 8 min according to the manufactures instructions (Ventana, Tuscon, Arizona, USA).

\section{In situ hybridization (ISH)}

To detect SARS-CoV-2 RNA, lung tissue sections were hybridized using specific probes for SARS-CoV-2 (ACD, Newark, CA, USA) followed by the RNAscope 2.5 HD Detection Kit Red from ACD (Newark, CA, USA) according to the manufacturer's protocol. 


\section{Determination of viral titers and viral RNA levels}

Homogenization of organs was performed in $1 \mathrm{ml} 1 \mathrm{x}$ PBS with 5 sterile, stainless steel beads (Ø $2 \mathrm{~mm}$, Retsch) at $30 \mathrm{~Hz}$ for $10 \mathrm{~min}$ in the mixer mill MM400 (Retsch).

For determination of the viral titer, the tissue homogenates were titrated on VeroE6 cells in 10fold serial dilutions for $30 \mathrm{~min}$ at $37^{\circ} \mathrm{C}$ and overlaid with MEM (Sigma-Aldrich) supplemented with $0,2 \% \mathrm{BSA}, 1 \% \mathrm{~L}$-glutamine, $1 \%$ penicillin-streptomycin, $1 \mu \mathrm{g} \mathrm{ml}^{-1} \mathrm{~L}$-1-tosylamido-2phenylethyl chloromethyl ketone (TPCK) treated trypsin (Sigma-Aldrich) and 1,25\% Avicel. After 72 hours p.i., cells were washed with 1x PBS, fixed with 4\% paraformaldehyde and the plaques were visualized by crystal violet staining. Viral RNA was isolated from homogenized organs using the QIAamp Viral RNA Mini Kit (QIAGEN) according to the manufacturer's instructions. SARS-CoV-2 RNA levels were then determined by quantitative reverse transcription real-time PCR (qRT-PCR) using the RealStar® SARS-CoV-2 RT-PCR Kit RUO (Altona Diagnostics). An internal control provided by the kit was used as a sample preparation control as well as an extended dry spin step for $10 \mathrm{~min}$ at $17,000 \mathrm{~g}$ at room temperature.

\section{Measurement of hormone and cytokine/chemokine levels}

Testosterone levels were measured in plasma samples using a custom-made MILLIPLEX MAP Multi-Species Hormone Magnetic Bead Panel (Merck), according to the manufacturer's instructions in a Bio-Plex 200 System with high-throughput fluidics (HTF; Bio-Rad). Estradiol levels (Calbiotech) in plasma samples were analyzed by ELISA following the manufacturer's instructions. ELISAs were measured on an Infinite M Nano ELISA microplate reader (Tecan). Female hamsters in diestrus were excluded from estradiol anaylsis.

A panel of 13 cytokines and chemokines (eotaxin, macrophage inflammatory protein $1 \alpha$ and $1 \beta$ (MIP-1 $\alpha,-1 \beta)$, interferon- $\gamma$ (IFN- $\gamma$ ), interleukin-2 (IL-2), interleukin-6 (IL-6), interleukin-10

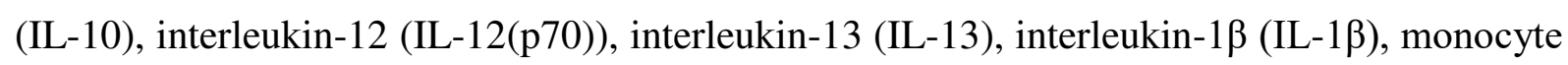
chemoattractant protein-1 (MCP-1), tumor necrosis factor- $\alpha$ (TNF- $\alpha$ ), and vascular endothelial growth factor (VEGF)) was measured in homogenized lungs using a custom-made Bio-Plex Pro $^{\text {TM }}$ Mouse Cytokine multiplex (Bio-Rad) according to the manufacturer's instructions in a Bio-Plex 200 System with high-throughput fluidics (HTF; Bio-Rad).

\section{Infection of Calu-3 cells with respiratory viruses}

Calu-3 cells (ATCC HTB-55) were seeded in 6-well plates. After 24 hours Calu-3 cells were infected with SARS-CoV, SARS-CoV-2 or H1N1 at a multiplicity of infection (MOI) of 1 in $500 \mu 1$ DMEM with $1 \%$ penicillin-streptomycin, $1 \%$ L-glutamine and 2\% FBS or DMEM with 
$1 \%$ penicillin-streptomycin, $1 \% \mathrm{~L}$-glutamine and $0.2 \% \mathrm{BSA}$, respectively, for $45 \mathrm{~min}$ at $37^{\circ} \mathrm{C}$. After washing twice with 1x PBS, $2 \mathrm{ml}$ of infection medium DMEM with $1 \%$ penicillinstreptomycin, $1 \%$ L-glutamine and $2 \%$ FBS or DMEM with containing $1 \%$ penicillinstreptomycin, $1 \%$ L-glutamine, $0.1 \%$ FBS and $1 \mu \mathrm{g} / \mathrm{ml}$ TPCK-treated Trypsin (Sigma-Aldrich $\mathrm{GmbH})$ were added and the cells were incubated for 24 hours at $37^{\circ} \mathrm{C}$.

\section{RNA isolation}

RNAprotect-fixed tissues were homogenized in $700 \mu 1$ lysis buffer RL with 5 sterile, stainless steel beads (Ø $2 \mathrm{~mm}$, Retch) at $30 \mathrm{~Hz}$ for $10 \mathrm{~min}$ in the mixer mill MM400 (Retsch). Calu-3 cells were lysed in $1 \mathrm{ml}$ TRIZOL (Invitrogen) per well, incubated for $5 \mathrm{~min}$ at RT. The cell lysate were resuspended and transferred into a fresh tube. $250 \mu \mathrm{l}$ chloroform were added to each sample, vortexted for $30 \mathrm{sec}$ and subsequently centrifuged for $15 \mathrm{~min}$ at $13,000 \mathrm{~g}$.

Total RNA was isolated from homogenized tissues and the aqueous phase of the lysed Calu-3 cells using the innuPREP RNA Mini Kit 2.0 (Analytik Jena) according to the manufacturer's instructions with an additional on column DNase I treatment using the RNase-free DNase Set (QIAGEN).

Total RNA from formalin-fixed, paraffin-embedded human lung tissue sections was purified using the RNeasy ${ }^{@}$ FFPE Kit (Qiagen) according to the manufacturer's instructions.

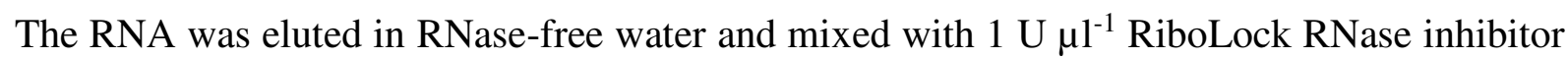
(Thermo Fisher Scientific).

\section{Determination of mRNA expression levels by real-time quantitative PCR (RT-qPCR)} The cDNA synthesis was performed using random nonamer primers (Gene Link, pd(N)9, final concentration: $5 \mu \mathrm{M}$ ), SuperScript III Reverse Transcriptase (Thermo Fisher Scientific) according to the manufacturer's instructions and $2 \mu \mathrm{g}$ total RNA. The cDNA was generated using the GeneAmp PCR System 9700 (Applied Biosystems; cycle: $25^{\circ} \mathrm{C}$ for $5 \mathrm{~min}, 50^{\circ} \mathrm{C}$ for $60 \mathrm{~min}, 70^{\circ} \mathrm{C}$ for $15 \mathrm{~min}, 4^{\circ} \mathrm{C}$ hold). Reactions were set up with PCR grade Water (Roche) in LightCycler® 480 Multiwell Plate 96 Reaction Plates (Roche). Briefly, $2 \mu 1$ of cDNA template were added to $10 \mu \mathrm{l}$ FastStart Essential DNA Green Master (Roche) and $300 \mathrm{nM}$ of forward and reverse primer, respectively. RT-qPCR runs were conducted using LightCycler® 96 RealTime PCR System (Roche) with endpoint fluorescence detection: $10 \mathrm{~min}$ at $95{ }^{\circ} \mathrm{C}$ and 45 amplification cycles $\left(15 \mathrm{~s}\right.$ at $95^{\circ} \mathrm{C}, 10 \mathrm{~s}$ at $65^{\circ} \mathrm{C}$ and $20 \mathrm{~s}$ at $\left.72{ }^{\circ} \mathrm{C}\right)$. Analysis was performed in duplicates for each gene. Negative controls and sample without reverse transcriptase were included to detect contaminations. 
Relative expression values were determined using a modified $\mathrm{E}^{-\Delta \Delta \mathrm{Ct}}$ method. Rn-values were exported from the LightCycler® 96 Software v1.1.0.1320 (Roche) to Microsoft Office Excel 2016 and $\mathrm{N}_{0}$-value for the starting concentration of the transcript in the original sample were obtained with LinReg PCR Software v2018.0 (Ruijter et al. 2009). The averaged $\mathrm{N}_{0}$-value of the CYP19A1 gene was then normalized with the average $\mathrm{N}_{0}$-value for HPRT ( $\mathrm{N}_{0(\mathrm{HPRT})}$ ) or RPL32 $\left(\mathrm{N}_{0(\mathrm{RPL} 32)}\right)$ of the respective sample. The relative $\mathrm{N}_{0(\text { CYP19A1) }} / \mathrm{N}_{0 \text { (HPRT) }}$ or

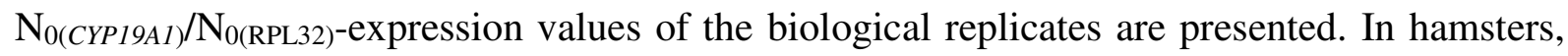
HPRT (Hypoxanthin phosphoribosyltransferase 1) was used as a reference gene for analyses of mRNA expression levels in the lung as well as in the brain and YWHAZ (Tyrosine 3Monooxygenase/Tryptophan 5-Monooxygenase Activation Protein Zeta) was used as a reference gene for analyses of mRNA expression levels in the gonads. In human samples, RPL32 (ribosomal protein L32) was used as a reference gene for analyses of mRNA expression levels in the lung. The following primer sequences were used for RT-qPCR: GTGATGGCCTCCCATCTCTT-3'; YWHAZ forward 5'-AGCCTTTGTCTGCCTCACTC3', reverse 5'- AAGTTAACTGGCTCTACTCCCC-3', ESR- $\alpha$ forward 5'TGCCCTACTACCTGGAGAATGA-3', reverse 5'-TGGTAGCCAGAGGCGTAGT-3'; ESRACGCCGGTTCTTGTCTATGG-3'; AR forward 5'-GGCCTTACCTGGATTCCGC-3', CCCTTGTACTGGGCTTCGTT-3', reverse 5'-CTGCTCCCAAAGCTACACCA-3'; TCTTTCAAGTCCTTGGCGGAT-3'; $5 \alpha$-reductase forward

5 ACCCGGGGAAACAGGATACA-3', reverse 5' - GAACAGGGCAAACACTCCAC-3'; 17ßHSD forward 5'-TGTGAATGTGGTCGGGACG-3', reverse 5 TGGAATGGCAGCCCCATCAA-3'; RPL32 (human) forward 5 GAAGTTCCTGGTCCACAACG-3', reverse 5'-GCGATCTCGGCACAGTAAG-3'; CYP19A1 (human) forward 5'-CGGCCTTGTTCGTATGGTCA-3', reverse 5'CAGAAGGGTCAACACGTCCA-3'.

\section{Differential gene expression analysis by stranded mRNA-Seq}

\section{Preparation and sequencing of cDNA libraries}

Preparation of stranded cDNA libraries was performed with Illumina Stranded mRNA Prep, 
Sequencing of the prepared stranded cDNA libraries was performed on an Illumina NextSeq 550 platform (Illumina Inc., San Diego, U.S.A.). In total, 30 cDNA libraries were paired-end sequenced in rapid mode with $2 \times 75$ cycles in two runs. Data analysis and base calling were accomplished with an in-house software platform as recently described (Wibberg et al., 2016). In total, $103 \mathrm{~Gb}$ sequence data were obtained for the 30 cDNA libraries, with an average of about $3.3 \mathrm{~Gb}$ (lowest $2.8 \mathrm{~Gb}$ ) per library. The sequencing raw data for all libraries has been made available on the EBI ArrayExpress server (www.ebi.ac.uk/arrayexpress), with the accession number E-MTAB-11162.

\section{Transcript mapping and differential gene expression analysis}

Read mapping and analyses were carried out as described recently with modifications ${ }^{55,56}$. In brief, the obtained reads were quality filtered (> Q30), trimmed and subsequently mapped to the 2.0 version of the Mesocricetus auratus (Syrian golden hamster) genome [RefSeq: JAFVMI010000001:JAFVMI010007057] using HISAT2 v.2.1.0 ${ }^{57}$ with default settings. Two mismatches were allowed to handle possible sequencing errors and allelic variants of the diploid genome. For transcript abundance analysis, resulting datasets were imported into LibiNorm 58 producing files that contain read count information, the normalized expression for each gene, calculated as TPM (Transcripts Per Million) values, with the RNA length used having been adjusted based on the bias that was identified for RNA of that length. In total, nine differential expression analyses with five replicates per groups were performed with DESeq2 ${ }^{59}$. An overview of the comparisons can be found in Table S1. For DESeq2 calculations, the genes were counted as differentially expressed with an adjusted P-value of less than 0.05 and a minimum fold change of 1 or more. Gene Ontology (GO) term enrichment was performed with GOrilla ${ }^{60}$ including all genes with a P-value of less than 0.05 and a minimum fold change of 1 or more (Table S2).

\section{GEN-COVID cohort, clinical classification and association}

The post-Mendelian model using an integrated polygenic score to predict COVID-19 disease severity was used with a Boolean representation of whole exome sequencing (WES) data of the GEN-COVID cohort ${ }^{35-38}$. The model was established previously with a set of least absolute shrinkage and selection operator (LASSO) logistic regression models trained and tested in multiple independent cohorts to identify gene variations contributing to COVID-19 severity. Patients of the GEN-COVID cohort were graded using the modified version of WHO COVID19 Outcome Scale used for severity grading, as follows: 5. death; 4. hospitalized receiving 
invasive mechanical ventilation; 3 . hospitalized, receiving continuous positive airway pressure (CPAP) or bilevel positive airway pressure (BiPAP) ventilation; 2. hospitalized, receiving lowflow supplemental oxygen; 1 . hospitalized, not receiving supplemental oxygen; and 0 . not hospitalized ${ }^{61}$. In order to obtain a clinical classification as independent as possible from age and sex, an Ordered Logistic Regression (OLR) model was used. Separately for the male and female cohorts, two OLR models were fitted using age to predict the ordinal grading $(0,1,2$, $3,4,5)$ dependent variable. Then, each patient was assigned a clinical classification: equal to 0 (milder), if the actual patient grading was below the one predicted by the OLR; or equal to 1 (more severe), if the grading was above the OLR prediction. The patients with a predicted grading equal to the actual grading were excluded from the following analyses in order to compare patients where the genetic contribution towards the more severe/milder phenotype is expected to be more relevant. In order to evaluate the significance of the association between Thr201Met CYP19A1 variant and COVID-19 severity, the Chi-Square Test was used.

\section{Data analysis}

All data were analyzed with Microsoft Office Excel 2016 and the Prism software (GraphPad, 9.0.1) using ordinary one-way analysis of variance (ANOVA) followed by Tukey's multiple comparisons test, ordinary two-way ANOVA followed by uncorrected Fisher's LSD test or Mann-Whitney test as indicated in the respective legends. Statistical significance was defined as $\mathrm{p} \leq 0.05(* \mathrm{p} \leq 0.05, * * \mathrm{p}<0.01, * * * \mathrm{p}<0.001)$. Additionally, ELISA data were evaluated using the "Four Parameter Logistic Curve" online data analysis tool, MyAssays Ltd., 25 th October 2012, http://www.myassays.com/four-parameter-logistic-curve.assay.

\section{Author contributions}

GG designed the study and wrote the manuscript. SSB, SB, NMK and BS performed all animal experiments, analyzed the data and revised the manuscript. FS, MZ, TB, HJ, ZM, KB, VPdR $\mathrm{MvKB}, \mathrm{CS}$ and $\mathrm{VKB}$ assisted with the animal experiments and performed molecular analysis. NMK, SSB, BS, SB, MW and SKE performed and/or interpreted data obtained from lung function experiments. $\mathrm{GB}, \mathrm{KB}, \mathrm{MdR}, \mathrm{DS}$ and $\mathrm{WB}$ performed histopathological and immunohistochemical analysis of the animal organs. TB, DW and JK performed RNA-seq and transcriptomic data analysis. MS, DJ, AN and SK provided clinical samples from COVID-19 patients for SARS-CoV-2 virus isolation. KK, MS, FH, SK, PL, BO, DvR, JvdT analyzed the lungs of fatal COVID-19 cases. AR, EF and CF analyzed human-genome wide data. All authors approved the manuscript. 


\section{Acknowledgment}

839 This study is dedicated to the memory of my academic mentor Hans Dieter Klenk $(* 25.06 .1938$,

840

841

842

843

844

845

846

847

848

849

850

851

852

853

854

855

856

857

858

859

860

861

862

863

$\dagger$ 01.06.2021). A pioneer in influenza research on interspecies transmission. He will be dearly missed.

This study was supported by a rapid response grant from the Federal Ministry of Health (BMG; ZMV I 1-2520COR501) and the Federal Ministry of Education and Research (BMBF; project no. 03COV06B) to G.G. W.B. received support by the BMBF project entitled RAPID (Risk assessment in re-pandemic respiratory infectious diseases; 01KI1723G). WB and MvKB received funding by the Ministry of Science and Culture of Lower Saxony in Germany (14 76103-184 CORONA-15/20). KK receives funding of the Ministry of Science, Research and the Arts Baden-Württemberg and the Deutsche Herzstiftung for research on SARS-CoV-2. JT received support from ZonMW through a COVID-19 programme research grant (project no. 1043001201 0016). FH, PL, BO and AH were funded by the German Federal Ministry of Education and Research (BMBF: 01KX2021) as part of the DEFEAT PANDEMIcs project and report on grants from the Authorities for Social Welfare, Hamburg, Germany. We thank all staff of the Core Facility Small Animal Models of the Leibniz Institute of Experimental Virology, particularly Ursula Müller and Oliver Strauch for their excellent support with the golden hamster model. We thank the technical staff of the CeBiTec Technology Platform Genomics, particularly Eva Schulte-Bernd, Yvonne Kutter and Katharina Hanuschka for technical assistance. We thank Kristin Hartmann, Martin Müller, Anna Lüttjohann und Jenny Ruschinski from the Leibniz Institute for Experimental Virology in Hamburg for technical assistance.

This study is part of the GEN-COVID Multicenter Study, https://sites.google.com/dbm.unisi.it/gen-covid, the Italian multicenter study aimed at identifying the COVID-19 host genetic bases. Specimens were provided by the COVID-19 Biobank of Siena, which is part of the Genetic Biobank of Siena, member of BBMRI-IT, of Telethon Network of Genetic Biobanks (project no. GTB18001), of EuroBioBank and of DConnect. We thank the CINECA consortium for providing computational resources and the Network for Italian Genomes (NIG) http://www.nig.cineca.it for its support. We thank private donors for the support provided to A.R. (Department of Medical Biotechnologies, University of Siena) for the COVID-19 host genetics research project (D.L n.18 of March 17, 2020). We 
871 also thank the COVID-19 Host Genetics Initiative (https://www.covid19hg.org/). This work 872 was funded by MIUR project "Dipartimenti di Eccellenza 2018-2020" to Department of 873 Medical Biotechnologies University of Siena, Italy (Italian D.L. n.18 March 17, 2020); private 874 donors for COVID-19 research and Charity fund 2020 from Intesa San Paolo dedicated to the 875 project N. B/2020/0119 "Identificazione delle basi genetiche determinanti la variabilità clinica 876 della risposta a COVID-19 nella popolazione italiana”. Part of this research project is also 877 funded by a Tuscany Region Bando Ricerca COVID-19 Toscana grant to the Azienda 878 Ospedaliero Universitaria Senese (CUP I49C20000280002), by the Italian Ministry of 879 University and Research within the Bando FISR 2020 in COVID-19 and by the Istituto Buddista 880 Italiano Soka Gakkai within the project PAT-COVID: Host Genetics and Pathogenetic 881 Mechanisms of COVID-19” (ID n. 2020-2016_RIC_3).

882

\section{Competing interest statement}

884 The authors declare no competing interests. 


\section{Figure Legends}

Figure 1. Lung plethysmography in SARS-CoV-2 infected male and female golden hamsters.

Whole-body plethysmography in SARS-CoV-2 infected or control (PBS) treated male (A,C,E,G,I) and female hamsters (B,D,F,H, J) at the indicated time points ( $n=10$; day 0 p.i. $n$ = 12): Tidal volume $(A, B)$, frequence $(C, D)$, EF50 $(E, F)$, peak inspiratory flow $(\mathrm{G}, \mathrm{H})$, peak expiratory flow $(\mathrm{I}, \mathrm{J})$. Values are shown as means; error bars are shown as SD. Statistical significance was assessed by Mann-Whitney test $(* \mathrm{p} \leq 0.05, * * \mathrm{p}<0.01, * * * \mathrm{p}<0.001)$.

\section{Figure 2. Sex hormones in SARS-CoV-2 infected male and female golden hamsters.}

(A-D) In male hamsters, testosterone (A), estradiol (C) and virus lung titers were measured at the indicated time points in SARS-CoV-2 $(n=5$; day 3 p.i. $n=10)$ infected or control treated (PBS $(n=5$; day 3 p.i. $n=10)$, Poly(I:C) $(n=5$; day 3 p.i. $n=10))$ animals. Confidence intervals are shown for difference of mean between SARS-CoV-2 infected and PBS treated groups regarding sex hormone levels testosterone (B) or estradiol (D) and virus titers in the lung. (E$\mathrm{H})$ In female hamsters, testosterone $(\mathrm{E})$, estradiol $(\mathrm{G})$ and virus lung titers were measured at the indicated time points in SARS-CoV-2 $(n=5$; day 3 p.i. $n=8-10)$ infected or control treated (PBS $(n=5$; day 3 p.i. $n=5-10)$, Poly(I:C) $((n=5$; day 3 p.i. $n=8-10))$ animals. Confidence intervals are shown for difference of mean between SARS-CoV-2 infected and PBS treated groups regarding sex hormone levels testosterone $(\mathrm{F})$ or estradiol $(\mathrm{H})$ and virus titers in the lung. Values are shown as means; error bars are shown as SD. Statistical significance was assessed by one-way ANOVA $(* p \leq 0.05, * * p<0.01, * * * p<0.001)$.

Figure 3. CYP19A1 expression in the lungs of SARS-CoV-2 infected male golden hamsters.

(A) Schematic overview of binding sites for transcription factors in the four promoter regions (P1, P2, P3, and P4) of the CYP19A1 gene. (B) Quantification of CYP19A1 expressing tissue in the lungs of control treated (PBS) male and female golden hamsters $(n=5)$. Statistical significance was assessed by Mann-Whitney test $(* * \mathrm{p}<0.01)$. (C-E) CYP19A1 mRNA expression levels measured at day $3(n=10)(\mathrm{C}), 6(n=5)(\mathrm{D})$ and $14(n=4-5)(\mathrm{E})$ p.i. in the lungs of SARS-CoV-2 infected and control treated (PBS, Poly(I:C)) male golden hamsters. Relative CYP19A1 mRNA expression values in PBS treated hamsters were set to 1 after normalization against HPRT. (F-I) Correlation analyses of MIP-1 $\alpha$ (F), MIP-1 $\beta$ (G), eotaxin 
(H) or VEGF (I) protein expression levels with CYP19A1 mRNA expression levels in SARS-

920 CoV-2 infected and control treated (PBS, Poly(I:C)) male golden hamsters at day 3 p.i. $(n=$

921

922

923

924

925

926

927

928

929

930

931

932

933

934

935

936

937

938

939

940

941

942

943

944

945

946

947

948

949

950

951
10). (J-K) Correlation analyses of testosterone (J) or estradiol (K) levels with CYP19A1 mRNA expression levels in SARS-CoV-2 infected and control treated (PBS, Poly(I:C)) male golden hamsters at day 3 p.i. $(n=10)$. (L) CYP19A1 mRNA expression levels detected in Calu-3 cells infected with H1N1 influenza A virus, SARS-CoV and SARS-CoV-2 (MOI=1) at 24 h p.i.. Relative CYP19A1 mRNA expression values in PBS treated cells were set to 1 after normalization against RPL32. Shown is one representative experiment of at least three independent experiments in triplicates. (M-P) Estrogen receptor $\alpha(\mathrm{ESR}-\alpha)(\mathrm{M})$, estrogen receptor $\beta(\mathrm{ESR}-\beta)(\mathrm{N})$, androgen receptor $(\mathrm{AR})(\mathrm{O})$ and zinc transporter 9 (ZIP-9) (P) mRNA expression levels measured in the lungs of SARS-CoV-2 infected and control treated (PBS, Poly(I:C)) male golden hamsters at day 3 p.i. $(n=5$, male Poly(I:C): $n=4)$. (C-P) Values are shown as means; error bars are shown as SD. Statistical significance was assessed by one-way ANOVA $(* \mathrm{p} \leq 0.05, * * * \mathrm{p}<0.001)$. Simple linear regression was used to determine statistical dependent variables $(\mathrm{F}-\mathrm{K})$.

\section{Figure 4. Lung plethysmography and pathology of SARS-CoV-2 infected hamsters} treated with the CYP19A1 inhibitor letrozole.

(A-D, H-K) Whole-body plethysmography in control (PBS) or SARS-CoV-2 infected male (AD) and female (H-K) golden hamsters, treated either with placebo or letrozole:Ttidal volume $(\mathrm{A}, \mathrm{H})$, frequence $(\mathrm{B}, \mathrm{I}), \mathrm{EF} 50(\mathrm{C}, \mathrm{J})$, inspiratory time $(\mathrm{D}, \mathrm{K})(n=5-7$; day 0 p.i. $n=12)$. $(\mathrm{E}, \mathrm{L})$ HE staining of lung sections from control (PBS) or SARS-CoV-2 infected male (E) and female (L) golden hamsters, treated either with placebo or letrozole, at $21 \mathrm{~d}$ p.i. Scale bar, $200 \mu \mathrm{m}(n$ = 5-7 per group). (F,M) Collagen staining with Sirius Red of lung sections from control (PBS) or SARS-CoV-2 infected male (F) and female (M) golden hamsters, treated either with placebo or letrozole, at $21 \mathrm{~d}$ p.i. Scale bar, $100 \mu \mathrm{m}$ ( $n=5-7$ per group). (G, N) Weight loss of control (PBS) or SARS-CoV-2 infected male (G) and female (N) golden hamsters, treated either with placebo or letrozole, over a time course of 21 days ( $n=5-7$ per group). Values are shown as means; error bars are shown as SD. Statistical significance was assessed by Mann-Whitney test or one-way ANOVA $(* * \mathrm{p}<0.01, * * * \mathrm{p}<0.001)$. 
Figure 5. Genome-wide transcriptome analysis in the lungs of SARS-CoV-2 infected hamsters treated with the CYP19A1 inhibitor letrozole.

The lung expression profiles of male and female golden hamsters, either non infected controls (PBS) or infected with SARS-CoV-2- or treated with placebo or with the CYP19A1 inhibitor letrozole were compared and are shown as ratio/intensity scatter plots (M/A-plot [M-value = $\log _{2}$ fold change; A-value $=\log _{2}$ base mean]). (A) Lung transcriptomes of SARS-CoV-2 infected and placebo treated male golden hamsters compared to control (PBS) and placebo treated male golden hamsters. (B) Lung transcriptomes of SARS-CoV-2 infected and placebo treated female golden hamsters compared to control (PBS) and placebo treated female hamsters. (C) Comparison of lung transcriptomes of SARS-CoV-2 infected male golden hamsters, treated either with letrozole or placebo. (D) Lung transcriptomes of SARS-CoV-2 infected and letrozole treated female golden hamsters compared to SARS-CoV-2 infected and placebo treated female golden hamsters. (E) Heatmap depicting normalized expression of differentially expressed genes in the lung of male and female golden hamsters $21 \mathrm{~d}$ p.i., clustered according to their expression profiles (cut-off $\log 2$ fold change $\geq 1$ or $\leq-1$, with an adjusted $p$-value $\leq 0.05$ ). (F-G) Significant differentially expressed gene in the lung of control (PBS) or SARS-CoV-2 infected male $(\mathrm{F})$ and female $(\mathrm{G})$ golden hamsters, treated either with placebo or letrozole. $(\mathrm{H})$ GO-term enrichment (biological process) of genes that were significantly downregulated in letrozole treated SARS-CoV-2 infected male golden hamsters compared to the placebo control group.

Figure 6. Association analysis of CYP19A1 gene variation in whole exome sequencing data of the GEN-COVID cohort.

(A) Characteristics and COVID-19 severity grading of the GEN-COVID cohort used to understand if variants of the CYP19A1 gene were associated with COVID-19 severity in humans. (B) Ordered Logistic Regression (OLR) model in males, fitted using age to predict the ordinal grading $(0,1,2,3,4,5)$ dependent variable. On the $\mathrm{Y}$ axis, the grading according to patients' treatment is reported. On the X axis, age is reported. Red dots represent subjects falling above the expected treatment outcomes according to age (hence considered severe), blue dots are subjects falling below the expected treatment outcomes according to age (hence considered mild) and black dots are subjects matching the expected treatment outcomes according to age (hence considered intermediate. (C) Pie chart representing the number of male patients falling into "milder", "more severe" and "predicted by sex and age" categories. (D) Ordered Logistic Regression (OLR) model in females, as described in (B). (E) Pie chart representing the number 
986

987

988

989

990

991

992

993

994

995

996

997

998

999

1000

1001

1002

1003

1004

1005

1006

1007

1008

1009

1010

1011

1012

1013

1014

1015

of female patients falling into "milder", "more severe" and "predicted by sex and age" categories. (F) Association analysis between Thr201Met CYP19A1 and COVID-19 severity was performed by Chi-Square Test separately for males and females patients. A significant association was found in males cohort. (G) Forest plot summarizing the association results in males and females.

\section{Figure 7. CYP19A1 expression in the lungs of fatal COVID-19 cases.}

(A) Detection of CYP19A1 protein (immunohistochemistry) and SARS-CoV-2 RNA (in situ hybridization) in the lungs of deceased male and female patients with COVID-19 or nonCOVID-19 controls (representative pictures are shown) from the University Hospital Tübingen. The consecutive tissue sections reveal that CYP19A1 positive cells, most likely macrophages, are infected with SARS-CoV-2. Scale bar, $100 \mu \mathrm{m}$. (B) Detection of CYP19A1 and SARSCoV-2 NP protein expression by immunohistochemistry of lungs from deceased male and female COVID-19 patients or non-COVID-19 controls (representative pictures are shown) from the University Hospital Hamburg-Eppendorf. Scale bar, $100 \mu \mathrm{m}$. (C) Detection of CYP19A1 protein expression by immunohistochemistry of lungs from deceased male COVID19 patients or non-COVID-19 controls (representative pictures are shown) from the Erasmus Medical Center Rotterdam. Scale bar, $50 \mu \mathrm{m}$. (D) CYP19A1 mRNA expression levels in the lungs from deceased male COVID-19 patients and non-COVID-19 controls at the University Hospital Tübingen (non-COVID-19: $n=8$, COVID-19: $n=9$ ). (E) CYP19A1 mRNA expression levels in the lungs from deceased male COVID-19 patients and non-COVID-19 controls at the University Hospital Hamburg-Eppendorf (non-COVID-19: $n=3$, COVID-19: $n=12$ ). (E) CYP19A1 mRNA expression levels in the lungs from deceased male COVID-19 patients and non-COVID-19 controls at the Erasmus Medical Center Rotterdam (non-COVID-19: $n=6$, COVID-19: $n=11$ ). Relative CYP19A1 mRNA expression values in non-COVID-19 controls were set to 1 after normalization against RPL32 (D-F). Values are shown as means; error bars are shown as SD. Statistical significance was assessed by Mann-Whitney test $(* * \mathrm{p}<0.01$, $* * * \mathrm{p}<0.001)$. 


\section{Supplementary Figures}

Figure S1. Increased disease severity in SARS-CoV-2 infected male golden hamsters.

(A) Weight loss of male and female golden hamsters infected with SARS-CoV-2 $(n=10)$. Statistical significance was assessed by one-way ANOVA $\left({ }^{*} \mathrm{p} \leq 0.05, * * \mathrm{p}<0.01, * * * \mathrm{p}<0.001\right)$. (B) HE staining of lung sections from control (PBS) or SARS-CoV-2 infected male and female golden hamsters at $3 \mathrm{~d}$ p.i. Representative pictures are shown $(n=5)$. Scale bar, $100 \mu \mathrm{m}$. $(\mathrm{C}$, D) Collagen staining with Azan (C) and quantification (D) of lung sections from control (PBS) or SARS-CoV-2 infected male and female golden hamsters at day 14 p.i. $(n=4-5)$. Scale bar, $50 \mu \mathrm{m}$. Values are shown as means; error bars are shown as SD. Statistical significance was assessed by two-way ANOVA $(* \mathrm{p} \leq 0.05, * * \mathrm{p}<0.01, * * * \mathrm{p}<0.001)$.

Figure S2: Lung plethysmography in SARS-CoV-2 infected male and female golden hamsters.

Whole-body plethysmography assessment of lung function parameters in SARS-CoV-2 infected or control (PBS) treated male (A,C,E,G,I) and female hamsters (B,D,F,H) at the indicated time points $(n=10$; day 0 p.i. $n=12)$ : Minute volume $(\mathrm{A}, \mathrm{B})$, end inspiratory pause $(\mathrm{C}, \mathrm{D})$, end expiratory pause $(\mathrm{E}, \mathrm{F})$, inspiratory time $(\mathrm{G}, \mathrm{H})$ and expiratory time $(\mathrm{I}, \mathrm{J})$. Values are shown as means; error bars are shown as SD. Statistical significance was assessed by MannWhitney test $(* \mathrm{p} \leq 0.05, * * \mathrm{p}<0.01, * * * \mathrm{p}<0.001)$.

Figure S3: Virus replication and cytokine induction in SARS-CoV-2 infected male and female golden hamsters.

(A, B) Viral lung titers of infected male and female golden hamsters on day 3 (A) and 6 (B) p.i. $(n=5)$. (C-J) MIP- $1 \alpha$ at day $3(\mathrm{C})$ and $6(\mathrm{G})$ p.i. $(n=5)$, MIP-1 $\beta$ at day $3(\mathrm{D})$ and $6(\mathrm{H})$ p.i. $(n$ $=5$ ), eotaxin at day 3 (E) and 6 (I) p.i. ( $n=5$; male Poly(I:C): $n=4)$ and VEGF at day 3 (F) and $6(\mathrm{~J})$ p.i. ( $n=4$; SARS-CoV-2: $n=3)$ protein expression levels in SARS-CoV-2 infected male and female golden hamsters. Values are shown as means; error bars are shown as SD. Statistical significance was assessed by two-way ANOVA $\left({ }^{*} \mathrm{p} \leq 0.05, * * * \mathrm{p}<0.001\right)$.

Figure S4. CYP19A1 protein expression in the lungs of SARS-CoV-2 infected male and female golden hamsters.

(A, B) Immunohistochemistry of serial sections from the lungs of SARS-CoV-2 infected and control treated (PBS, Poly(I:C)) male (A) and female (B) golden hamsters for CYP19A1, 
SARS-CoV-2 NP, macrophages (CD204), endothelial cells (vWF and CD31), T cells (CD3) and smooth muscle actin (SMA). Representative pictures of each group are shown $(n=5)$. Scale bar, $100 \mu \mathrm{m}$. (C, D) Quantification of CYP19A1 expressing tissue in the lungs of SARS-CoV2 infected and control treated (PBS, Poly(I:C)) male (C) and female (D) golden hamsters $(n=$ 5). Data from PBS treated animals are also shown in Figure 3B. Statistical significance was assessed by one-way ANOVA $\left({ }^{*} \mathrm{p} \leq 0.05\right)$.

Figure S5. CYP19A1 mRNA expression in the lungs of SARS-CoV-2 infected female golden hamsters.

(A-C) CYP19A1 mRNA expression levels measured at day $3(n=10)(\mathrm{A}), 6(n=5)(\mathrm{B})$ and 14 $(n=4-5)(\mathrm{C})$ p.i. in the lungs of SARS-CoV-2 infected and control treated (PBS, Poly(I:C)) female golden hamsters. Relative CYP19A1 mRNA expression values in PBS treated hamsters were set to 1 after normalization against HPRT. (D-G) Correlation analyses of MIP-1 $\alpha$ (D), MIP-1 $\beta(\mathrm{E})$, eotaxin (F) or VEGF (G) protein expression levels with CYP19A1 mRNA expression levels in SARS-CoV-2 infected and control treated (PBS, Poly(I:C)) female golden hamsters at day 3 p.i. $(n=10)$. $(\mathrm{H}, \mathrm{I})$ Correlation analyses of testosterone $(\mathrm{H})$ or estradiol $(\mathrm{I})$ levels with CYP19A1 mRNA expression levels in SARS-CoV-2 infected and control treated (PBS, Poly(I:C)) female golden hamsters at day 3 p.i. $(n=10)$. (J-M) Estrogen receptor $\alpha$ (ESR$\alpha)(J)$, estrogen receptor $\beta($ ESR $\beta$ ) $(\mathrm{K})$, androgen receptor (AR) (L) and zinc transporter 9 (ZIP9) (M) mRNA expression levels measured in the lungs of SARS-CoV-2 infected and control treated (PBS, Poly(I:C)) female golden hamsters at day 3 p.i. $(n=5$, male Poly(I:C): $n$ =4). Values are shown as means; error bars are shown as SD. Statistical significance was assessed by one-way ANOVA $(* \mathrm{p} \leq 0.05, * * * \mathrm{p}<0.001)$ (A-C,J-M). Simple linear regression was used to determine statistical dependent variables (D-I).

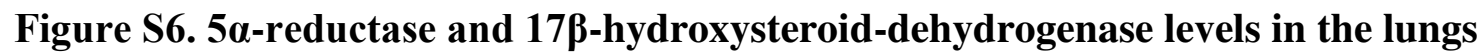
SARS-CoV-2 infected of male and female golden hamsters.

(A, C) 5 $\alpha$-reductase mRNA expression levels measured in the lungs of SARS-CoV-2 infected and control treated (PBS, Poly(I:C)) male (A) and female (C) golden hamsters at day 3 p.i.. (B, D) $17 \beta$-HSD mRNA expression levels detected in the lungs of SARS-CoV-2 infected and control treated (PBS, Poly(I:C)) male (B) and female (D) golden hamsters at day 3 p.i.. Values are shown as means; error bars are shown as $\mathrm{SD}(n=5$, male Poly(I:C): $n=4)$. Relative CYP19A1 mRNA expression values in PBS treated hamsters were set to 1 after normalization against HPRT. 
Figure S7. Virus titer and CYP19A1 expression in the gonads of SARS-CoV-2 infected male and female golden hamsters.

(A, E) Viral mRNA and virus titer detected in the testis of SARS-CoV-2 infected and control treated (PBS, Poly(I:C)) male (A) and ovaries of female (E) golden hamsters at day 3 d p.i. (n = 5). n.d., not detectable. (B, F) CYP19A1 mRNA expression levels measured in the testis of SARS-CoV-2 infected and control treated (PBS, Poly(I:C)) male (B) and ovaries of female (F) golden hamsters at day 3 p.i. $(n=5)$. Relative CYP19A1 mRNA expression values in PBS treated hamsters were set to 1 after normalization against YWHAZ. $(\mathrm{C}, \mathrm{G})$ Quantification of CYP19A1 protein expressing tissue in the testis of SARS-CoV-2 infected and control treated (PBS, Poly(I:C)) male (C) and ovaries of female (G) golden hamsters at day 3 p.i. $(n=5)$. (D, H) CYP19A1 protein expression detected by immunohistochemistry in the testis of SARSCoV-2 infected and control treated (PBS, Poly(I:C)) male (D) and ovaries of female (H) golden hamsters. Consecutive slides were also stained with HE. Representative pictures of each group are shown $(n=5)$. Scale bar, $100 \mu \mathrm{m}$. Values are shown as means; error bars are shown as SD. Statistical significance was assessed by one-way ANOVA $\left(* p \leq 0.05,{ }^{*} p<0.01\right)(A, B, E, F)$.

Figure S8. Virus titer and CYP19A1 expression in the brains of SARS-CoV-2 infected male and female golden hamsters.

(A,D) Viral titer detected in the brains of SARS-CoV-2 infected and control treated (PBS, Poly(I:C)) male (A) and female (D) golden hamsters at day 3 p.i. $(n=5)$. n.d., not detectable. (B, E) CYP19A1 mRNA expression levels measured in the brains of SARS-CoV-2 infected and control treated (PBS, Poly(I:C)) male (B) and female (E) golden hamsters at day 3 p.i. $(n=5)$. Relative CYP19A1 mRNA expression values in PBS treated hamsters were set to 1 after normalization against HPRT. (C,F) CYP19A1 protein expression detected by immunohistochemistry in the brains of SARS-CoV-2 infected and control treated (PBS, Poly(I:C)) male (C) and female (F) golden hamsters. Consecutive slides were also stained with HE. Representative pictures of each group are shown $(n=5)$. Scale bar, $100 \mu \mathrm{m}$. Values are shown as means; error bars are shown as $\mathrm{SD}(\mathrm{A}, \mathrm{B}, \mathrm{D}, \mathrm{E})$.

Figure S9. Sex hormone levels in SARS-CoV-2 infected hamsters treated with the CYP19A1 inhibitor letrozole.

(A) Timeline of experimental setup for letrozole therapy. Male and female golden hamsters were infected with SARS-CoV-2 and subjected to daily letrozole treatment from $3 \mathrm{~h}$ to $8 \mathrm{~d}$ p.i.. Body weight and lung function were monitored over a time course of 21 days. (B-E) 
Testosterone (B, D) and estradiol (C, E) levels were measured in control (PBS) or SARS-CoV2 infected male (B-C) and female (D-E) golden hamsters, treated either with placebo or letrozole, at 3 d p.i.. $(n=11)$. (F-I) AR (F), ZIP-9 (G), ESR- $\alpha(\mathrm{H})$ and ESR- $\beta$ (I) mRNA expression levels measured in the lungs of SARS-CoV-2 infected and control treated (PBS, Poly(I:C)) male golden hamsters at day 6 p.i. $(n=11)$. (J-M) AR (J), ZIP-9 (K), ESR- $\alpha(\mathrm{L})$ and ESR- $\beta$ (M) mRNA expression levels measured in the lungs of SARS-CoV-2 infected and control treated (PBS, Poly(I:C)) female golden hamsters at day 6 p.i. $(n=11)$. SARS-CoV-2 infected and placebo treated hamsters were set as 1 (F-M). Values are shown as means; error bars are shown as SD. Statistical significance was assessed by Mann-Whitney test $\left({ }^{*} p \leq 0.05\right)$.

Figure S10: Virus replication and cytokine induction in SARS-CoV-2 infected male and female golden hamsters treated with the CYP19A1 inhibitor letrozole.

(A,B) Viral lung titers of SARS-CoV-2 or control (PBS) infected male (A) and female (B) golden hamsters, treated either with placebo or letrozole on day 3 p.i. $(n=6)$. (C-J) Protein expression levels of MIP-1 $\alpha$ in male (C) and female (D), MIP-1 $\beta$ in male (E) and female (F), eotaxin in male (G) and female (H) and VEGF in male (I) and female (J) golden hamstersat day 3 p.i. $(n=6)$. Values are shown as means; error bars are shown as SD. Statistical significance was assessed by one-way ANOVA $(* * p<0.01, * * * p<0.001)$.

Figure S11. Lung plethysmography in PBS control infected male golden hamsters treated with the CYP19A1 inhibitor letrozole.

Whole-body plethysmography assessment of lung function parameters in control infected male hamsters, treated either with placebo or letrozole, at the indicated time points $(n=5-7$; day 0 p.i. $n=12$ ): Tidal volume (A), frequence (B), EF50 (C), peak inspiratory flow (D), peak expiratory flow $(E)$, minute volume $(F)$, end inspiratory pause $(G)$, end expiratory pause $(H)$, inspiratory time (I) and expiratory time (J). Values are shown as means; error bars are shown as $\mathrm{SD}$.

Figure S12. Lung plethysmography in PBS control infected female golden hamsters treated with the CYP19A1 inhibitor letrozole.

Whole-body plethysmography assessment of lung function parameters in control infected female hamsters, treated either with placebo or letrozole, at the indicated time points $(n=5-7$; day 0 p.i. $n=12$ ): tidal volume (A), frequence (B), EF50 (C), peak inspiratory flow (D), peak expiratory flow $(\mathrm{E})$, minute volume $(\mathrm{F})$, end inspiratory pause $(\mathrm{G})$, end expiratory pause $(\mathrm{H})$, 
1152 inspiratory time (I) and expiratory time (J). Values are shown as means; error bars are shown 1153 as SD.

1154

1155 Figure S13. Lung plethysmography in SARS-CoV-2 infected male and female golden 1156 hamsters treated with the CYP19A1 inhibitor letrozole.

1157 Whole-body plethysmography assessment of lung function parameters in SARS-CoV-2 1158 infected or control (PBS) infected male (A,C,E,G,I,K) and female hamsters (B,D,F,H,J,L), 1159 treated either with placebo or letrozole, at the indicated time points $(n=5-7$; day 0 p.i. $n=12)$ : 1160 Minute volume (A,B), end inspiratory pause (C,D), end expiratory pause (E,F), expiratory time $1161(\mathrm{G}, \mathrm{H})$, peak inspiratory flow $(\mathrm{I}, \mathrm{J})$ and peak expiratory flow $(\mathrm{K}, \mathrm{L})$. Values are shown as means; 1162 error bars are shown as SD. 
1165 Supplementary Tables

1166

1167 Table S1. Overview of RNAseq data.

1168

1169 Table S2. Gene ontology term enrichment for biological processes.

1170 
1. Dong E, Du H, Gardner L. An interactive web-based dashboard to track COVID-19 in real time. Lancet Infect Dis 2020;20:533-4.

2. Williamson EJ, Walker AJ, Bhaskaran K, et al. Factors associated with COVID-19-related death using OpenSAFELY. Nature 2020;584:430-6.

3. Clark A, Jit M, Warren-Gash C, et al. Global, regional, and national estimates of the population at increased risk of severe COVID-19 due to underlying health conditions in 2020: a modelling study. Lancet Glob Health 2020;8:e1003-e17.

4. Jin JM, Bai P, He W, et al. Gender Differences in Patients With COVID-19: Focus on Severity and Mortality. Front Public Health 2020;8:152.

5. Bhopal SS, Bhopal R. Sex differential in COVID-19 mortality varies markedly by age. Lancet 2020;396:532-3.

6. Goodman KE, Magder LS, Baghdadi JD, et al. Impact of Sex and Metabolic Comorbidities on COVID-19 Mortality Risk Across Age Groups: 66,646 Inpatients Across 613 U.S. Hospitals. Clin Infect Dis 2020.

7. Krieger N, Chen JT, Waterman PD. Excess mortality in men and women in Massachusetts during the COVID-19 pandemic. Lancet 2020;395:1829.

8. Haitao T, Vermunt JV, Abeykoon J, et al. COVID-19 and Sex Differences: Mechanisms and Biomarkers. Mayo Clin Proc 2020;95:2189-203.

9. Decaroli MC, Rochira V. Aging and sex hormones in males. Virulence 2017;8:545-70.

10. Dhindsa S, Zhang N, McPhaul MJ, et al. Association of Circulating Sex Hormones With Inflammation and Disease Severity in Patients With COVID-19. JAMA Netw Open 2021;4:e2111398.

11. Infante $\mathrm{M}$, Pieri $\mathrm{M}$, Lupisella $\mathrm{S}$, et al. Low testosterone levels and high estradiol to testosterone ratio are associated with hyperinflammatory state and mortality in hospitalized men with COVID-19. Eur Rev Med Pharmacol Sci 2021;25:5889-903.

12. Salonia A, Pontillo M, Capogrosso P, et al. Severely low testosterone in males with COVID-19: A case-control study. Andrology 2021;9:1043-52.

13. Schroeder M, Schaumburg B, Mueller Z, et al. High estradiol and low testosterone levels are associated with critical illness in male but not in female COVID-19 patients: a retrospective cohort study. Emerg Microbes Infect 2021;10:1807-18.

14. Wierman ME. Sex steroid effects at target tissues: mechanisms of action. Adv Physiol Educ 2007;31:26-33.

15. Townsend EA, Miller VM, Prakash YS. Sex differences and sex steroids in lung health and disease. Endocr Rev 2012;33:1-47.

16. Seaborn T, Simard M, Provost PR, Piedboeuf B, Tremblay Y. Sex hormone metabolism in lung development and maturation. Trends Endocrinol Metab 2010;21:729-38.

17. Wulfsohn NL, Politzer WM, Henrico JS. Testosterone Therapy in Bronchial Asthma. S Afr Med J 1964;38:170-2.

18. Fuentes N, Silveyra P. Endocrine regulation of lung disease and inflammation. Exp Biol Med (Maywood) 2018;243:1313-22.

19. Triebner K, Matulonga B, Johannessen A, et al. Menopause Is Associated with Accelerated Lung Function Decline. Am J Respir Crit Care Med 2017;195:1058-65.

20. Imai M, Iwatsuki-Horimoto K, Hatta M, et al. Syrian hamsters as a small animal model for SARSCoV-2 infection and countermeasure development. Proc Natl Acad Sci U S A 2020;117:16587-95.

21. Sia SF, Yan LM, Chin AWH, et al. Pathogenesis and transmission of SARS-CoV-2 in golden hamsters. Nature 2020;583:834-8.

22. Nathan L, Shi W, Dinh $\mathrm{H}$, et al. Testosterone inhibits early atherogenesis by conversion to estradiol: critical role of aromatase. Proc Natl Acad Sci U S A 2001;98:3589-93.

23. Diano S, Horvath TL, Mor G, et al. Aromatase and estrogen receptor immunoreactivity in the coronary arteries of monkeys and human subjects. Menopause 1999;6:21-8. 
24. Nebert DW, Wikvall K, Miller WL. Human cytochromes P450 in health and disease. Philos Trans R Soc Lond B Biol Sci 2013;368:20120431.

25. Enjuanes A, Garcia-Giralt N, Supervia A, et al. Functional analysis of the I.3, I.6, pll and I.4 promoters of CYP19 (aromatase) gene in human osteoblasts and their role in vitamin D and dexamethasone stimulation. Eur J Endocrinol 2005;153:981-8.

26. Kamat A, Graves KH, Smith ME, Richardson JA, Mendelson CR. A 500-bp region, approximately $40 \mathrm{~kb}$ upstream of the human CYP19 (aromatase) gene, mediates placenta-specific expression in transgenic mice. Proc Natl Acad Sci U S A 1999;96:4575-80.

27. Sebastian S, Takayama K, Shozu M, Bulun SE. Cloning and characterization of a novel endothelial promoter of the human CYP19 (aromatase P450) gene that is up-regulated in breast cancer tissue. Mol Endocrinol 2002;16:2243-54.

28. Toda K, Terashima M, Kawamoto T, et al. Structural and functional characterization of human aromatase P-450 gene. Eur J Biochem 1990;193:559-65.

29. Zhao Y, Mendelson CR, Simpson ER. Characterization of the sequences of the human CYP19 (aromatase) gene that mediate regulation by glucocorticoids in adipose stromal cells and fetal hepatocytes. Mol Endocrinol 1995;9:340-9.

30. Cisternas CD, Cabrera Zapata LE, Arevalo MA, Garcia-Segura LM, Cambiasso MJ. Regulation of aromatase expression in the anterior amygdala of the developing mouse brain depends on ERbeta and sex chromosome complement. Sci Rep 2017;7:5320.

31. Goss PE, Ingle JN, Martino S, et al. A randomized trial of letrozole in postmenopausal women after five years of tamoxifen therapy for early-stage breast cancer. N Engl J Med 2003;349:1793-802.

32. de Boer $H$, Verschoor L, Ruinemans-Koerts J, Jansen $M$. Letrozole normalizes serum testosterone in severely obese men with hypogonadotropic hypogonadism. Diabetes Obes Metab 2005;7:211-5.

33. Artigalas O, Vanni T, Hutz MH, Ashton-Prolla P, Schwartz IV. Influence of CYP19A1 polymorphisms on the treatment of breast cancer with aromatase inhibitors: a systematic review and meta-analysis. BMC Med 2015;13:139.

34. Beitelshees $\mathrm{AL}$, Johnson JA, Hames $\mathrm{ML}$, et al. Aromatase gene polymorphisms are associated with survival among patients with cardiovascular disease in a sex-specific manner. PLoS One 2010;5:e15180.

35. Initiative C-HG. Mapping the human genetic architecture of COVID-19. Nature 2021.

36. Daga S, Fallerini C, Baldassarri M, et al. Employing a systematic approach to biobanking and analyzing clinical and genetic data for advancing COVID-19 research. Eur J Hum Genet 2021;29:745-59. 37. Picchiotti N, Benetti E, Fallerini C, et al. Post-Mendelian genetic model in COVID-19. MedRxive 2021.

38. Fallerini C, Picchiotti N, Baldassarri M, et al. Common, low-frequency, rare, and ultra-rare coding variants contribute to COVID-19 severity. MedRxive 2021.

39. Payne EJ, Ingley E, Dick IM, Wilson SG, Bond CS, Prince RL. In vitro kinetic properties of the Thr201Met variant of human aromatase gene CYP19A1: functional responses to substrate and product inhibition and enzyme inhibitors. J Clin Endocrinol Metab 2009;94:2998-3002.

40. Salonia A, Pontillo M, Capogrosso P, et al. Testosterone in males with COVID-19: A 7-month cohort study. Andrology 2021.

41. Zickler M, Stanelle-Bertram S, Ehret S, et al. Replication of SARS-CoV-2 in Adipose Tissue Determines Organ and Systemic Lipid Metabolism in Hamsters and Humans. SSRN 2021.

42. Agudelo CW, Samaha G, Garcia-Arcos I. Alveolar lipids in pulmonary disease. A review. Lipids Health Dis 2020;19:122.

43. Gilliver SC, Ruckshanthi JP, Hardman MJ, Nakayama T, Ashcroft GS. Sex dimorphism in wound healing: the roles of sex steroids and macrophage migration inhibitory factor. Endocrinology 2008;149:5747-57.

44. Carey MA, Card JW, Voltz JW, Germolec DR, Korach KS, Zeldin DC. The impact of sex and sex hormones on lung physiology and disease: lessons from animal studies. Am J Physiol Lung Cell Mol Physiol 2007;293:L272-8. 
45. Cephus JY, Stier MT, Fuseini H, et al. Testosterone Attenuates Group 2 Innate Lymphoid CellMediated Airway Inflammation. Cell Rep 2017;21:2487-99.

46. Lenoir A, Fuertes E, Gomez-Real F, Leynaert B, van der Plaat DA, Jarvis D. Lung function changes over 8 years and testosterone markers in both sexes: UK Biobank. ERJ Open Res 2020;6.

47. Cohen PG. The hypogonadal-obesity cycle: role of aromatase in modulating the testosteroneestradiol shunt--a major factor in the genesis of morbid obesity. Med Hypotheses 1999;52:49-51.

48. Cohen PG. Obesity in men: the hypogonadal-estrogen receptor relationship and its effect on glucose homeostasis. Med Hypotheses 2008;70:358-60.

49. Arora P, Gudelsky G, Desai PB. Gender-based differences in brain and plasma pharmacokinetics of letrozole in sprague-dawley rats: Application of physiologically-based pharmacokinetic modeling to gain quantitative insights. PLoS One 2021;16:e0248579.

50. Jelovac D, Macedo L, Goloubeva OG, Handratta V, Brodie AM. Additive antitumor effect of aromatase inhibitor letrozole and antiestrogen fulvestrant in a postmenopausal breast cancer model. Cancer Res 2005;65:5439-44.

51. Liu XD, Xie L, Zhong Y, Li CX. Gender difference in letrozole pharmacokinetics in rats. Acta Pharmacol Sin 2000;21:680-4.

52. Sabnis G, Goloubeva O, Gilani R, Macedo L, Brodie A. Sensitivity to the aromatase inhibitor letrozole is prolonged after a "break" in treatment. Mol Cancer Ther 2010;9:46-56.

53. Wempe MF, Buchanan CM, Buchanan NL, et al. Pharmacokinetics of letrozole in male and female rats: influence of complexation with hydroxybutenyl-beta cyclodextrin. J Pharm Pharmacol 2007;59:795-802.

54. Bosmuller $\mathrm{H}$, Traxler S, Bitzer M, et al. The evolution of pulmonary pathology in fatal COVID-19 disease: an autopsy study with clinical correlation. Virchows Arch 2020;477:349-57.

55. Verwaaijen B, Wibberg D, Krober M, et al. The Rhizoctonia solani AG1-IB (isolate $7 / 3 / 14$ ) transcriptome during interaction with the host plant lettuce (Lactuca sativa L.). PLoS One 2017;12:e0177278.

56. Zrenner R, Genzel F, Verwaaijen B, Wibberg D, Grosch R. Necrotrophic lifestyle of Rhizoctonia solani AG3-PT during interaction with its host plant potato as revealed by transcriptome analysis. Sci Rep 2020;10:12574.

57. Kim D, Paggi JM, Park C, Bennett C, Salzberg SL. Graph-based genome alignment and genotyping with HISAT2 and HISAT-genotype. Nat Biotechnol 2019;37:907-15.

58. Dyer NP, Shahrezaei V, Hebenstreit D. LiBiNorm: an htseq-count analogue with improved normalisation of Smart-seq2 data and library preparation diagnostics. PeerJ 2019;7:e6222.

59. Love MI, Huber W, Anders S. Moderated estimation of fold change and dispersion for RNA-seq data with DESeq2. Genome Biol 2014;15:550.

60. Eden E, Navon R, Steinfeld I, Lipson D, Yakhini Z. GOrilla: a tool for discovery and visualization of enriched GO terms in ranked gene lists. BMC Bioinformatics 2009;10:48.

61. Synopsis. C-TT. WHO R\&D Blue Print. WHO R\&D Blue Print 2021. 


\section{Figures}

\section{Figure 1}

Lung plethysmography in SARS-CoV-2 infected male and female golden hamsters. Whole-body plethysmography in SARS-CoV-2 infected or control (PBS) treated male $(A, C, E, G, I)$ and female hamsters $(B, D, F, H, J)$ at the indicated time points $(n=10$; day 0 p.i. $n=12)$ : Tidal volume $(A, B)$, frequence $(C, D)$, EF50 $(E, F)$, peak inspiratory flow $(G, H)$, peak expiratory flow $(I, J)$. Values are shown as means; error bars are shown as SD. Statistical significance was assessed by Mann-Whitney test $\left({ }^{*} p \leq 0.05,{ }^{* *} p<0.01\right.$, $\star \star \star x<0.001)$. 
A

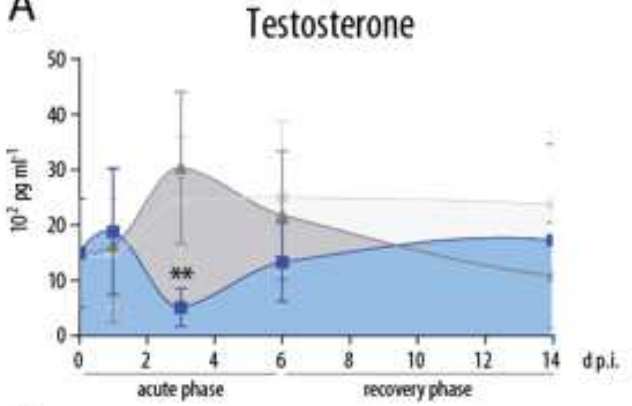

C

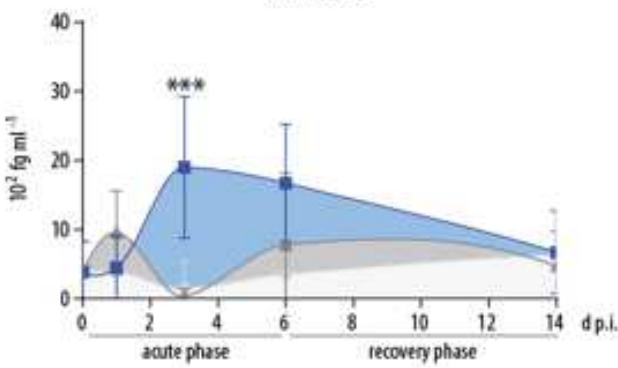

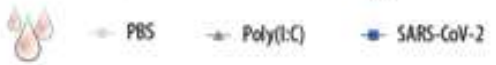

E

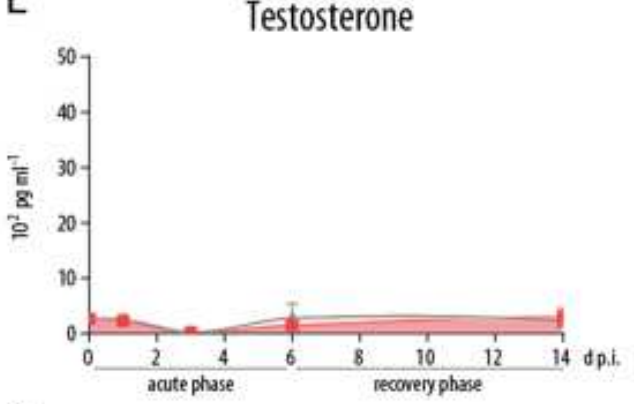

G

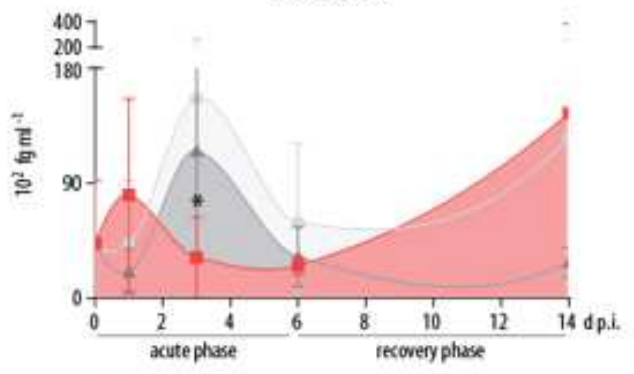

fys - PBS + Polytico $=$ SARS-COVV-2
B

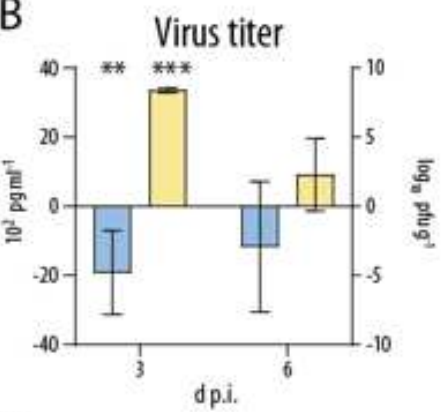

D

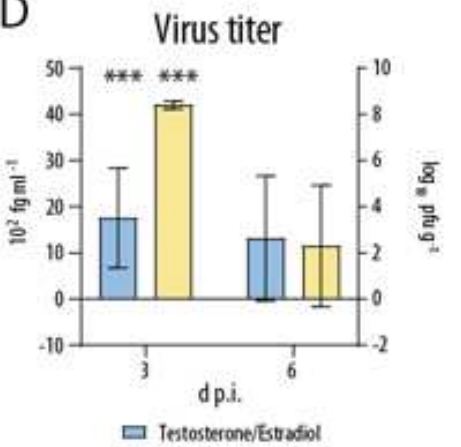

$\square$ SARS-CoV-2lung Titer

F

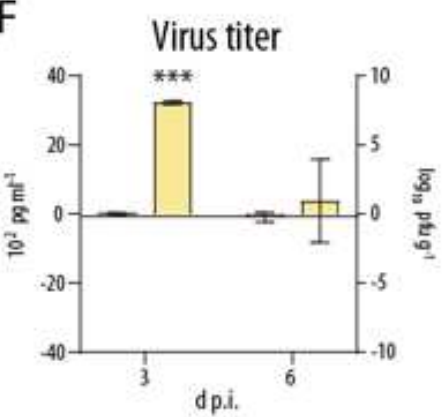

h

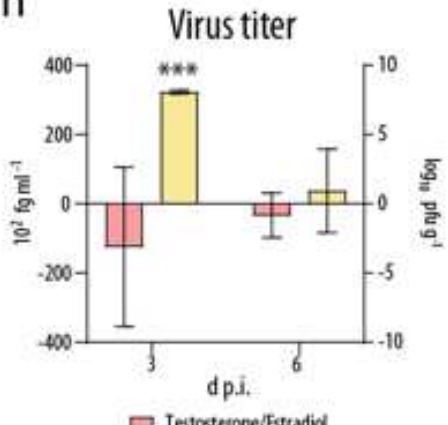

므 Testostecone/Estradial

口 SARS-COV-2 Lung Iiter

Figure 2

\section{Figure 2}

Sex hormones in SARS-CoV-2 infected male and female golden hamsters. (A-D) In male hamsters, testosterone (A), estradiol (C) and virus lung titers were measured at the indicated time points in SARSCoV-2 $(n=5$; day 3 p.i. $n=10)$ infected or control treated (PBS $(n=5$; day 3 p.i. $n=10)$, Poly $(l: C)(n=5$; day 3 p.i. $n=10)$ ) animals. Confidence intervals are shown for difference of mean between SARS-CoV-2 infected and PBS treated groups regarding sex hormone levels testosterone (B) or estradiol (D) and virus 
titers in the lung. $(\mathrm{E}-\mathrm{H})$ In female hamsters, testosterone $(\mathrm{E})$, estradiol $(\mathrm{G})$ and virus lung titers were measured at the indicated time points in SARS-CoV-2 $(n=5$; day 3 p.i. $n=8-10)$ infected or control treated (PBS $(n=5$; day 3 p.i. $n=5-10)$, Poly $(I: C)((n=5$; day 3 p.i. $n=8-10))$ animals. Confidence intervals are shown for difference of mean between SARS-CoV-2 infected and PBS treated groups regarding sex hormone levels testosterone $(\mathrm{F})$ or estradiol $(\mathrm{H})$ and virus titers in the lung. Values are shown as means; error bars are shown as SD. Statistical significance was assessed by one-way ANOVA ( ${ }^{*} p \leq 0.05$, $\star \star p<0.01, * \star \star p<0.001)$.

\section{Figure 3}

CYP19A1 expression in the lungs of SARS-CoV-2 infected male golden 909 hamsters. (A) Schematic overview of binding sites for transcription factors in the four promoter regions (P1, P2, P3, and P4) of the CYP19A1 gene. (B) Quantification of CYP19A1 expressing tissue 912 in the lungs of control treated (PBS) male and female golden hamsters $(n=5)$. Statistical significance was assessed by Mann-Whitney test $(\star \star p<0.01)$. (C-E) CYP19A1 mRNA expression levels measured at day $3(n=10)(C), 6(n=5)(D)$ and $14(n$ $=4-5)(E)$ p.i. in the lungs of SARS-CoV-2 infected and control treated (PBS, Poly $(\mathrm{I}: \mathrm{C})$ ) male golden hamsters. Relative CYP19A1 mRNA expression values in PBS treated hamsters were set to 1 after normalization against HPRT. (F-I) Correlation analyses of MIP-1a (F), MIP-1 $\beta(G)$, eotaxin (H) or VEGF (I) protein expression levels with CYP19A1 mRNA expression levels in SARS-CoV-2 infected and control treated (PBS, Poly $(\mathrm{I}: \mathrm{C}))$ male golden hamsters at day 3 p.i. $(\mathrm{n}=10)$. $(\mathrm{J}-\mathrm{K})$ Correlation analyses of testosterone $(\mathrm{J})$ or estradiol $(\mathrm{K})$ levels with CYP19A1 mRNA expression levels in SARS-CoV-2 infected and control treated (PBS, Poly $(\mathrm{I}: \mathrm{C}))$ male golden hamsters at day 3 p.i. $(\mathrm{n}=10)$. $(\mathrm{L})$ CYP19A1 mRNA expression levels detected in Calu-3 cells infected with H1N1 influenza A virus, SARS-CoV and SARS-CoV2 (MOI=1) at $24 \mathrm{~h}$ p.i.. Relative CYP19A1 mRNA expression values in PBS treated cells were set to 1 after normalization against RPL32. Shown is one representative experiment of at least three independent experiments in triplicates. (M-P) Estrogen receptor a (ESR-a) (M), estrogen receptor $\beta(E S R-\beta)(N)$, androgen receptor (AR) (O) and zinc transporter 9 (ZIP-9) (P) mRNA expression levels measured in the lungs of SARS-CoV-2 infected and control treated (PBS, Poly (I:C)) male golden hamsters at day 3 p.i. $(n=$ 5 , male Poly $(I: C): n=4)$. (C-P) Values are shown as means; error bars are shown as SD. Statistical significance was assessed by one-way ANOVA ( ${ }^{*} p \leq 0.05$, $\left.{ }^{\star \star *} p<0.001\right)$. Simple linear regression was used to determine statistical dependent variables (F-K).

\section{Figure 4}

Lung plethysmography and pathology of SARS-CoV-2 infected hamsters treated with the CYP19A1 inhibitor letrozole. (A-D, H-K) Whole-body plethysmography in control (PBS) or SARS-CoV-2 infected male $(A-D)$ and female $(H-K)$ golden hamsters, treated either with placebo or letrozole:Tidal volume $(A, H)$, frequence $(B, I), E F 50(C, J)$, inspiratory time $(D, K)(n=5-7$; day 0 p.i. $n=12)$. $(E, L) H E$ staining of lung sections from control (PBS) or SARS-CoV-2 infected male (E) and female (L) golden hamsters, treated 
either with placebo or letrozole, at $21 \mathrm{~d}$ p.i. Scale bar, $200 \mu \mathrm{m}$ ( $\mathrm{n}=5-7$ per group). (F,M) Collagen staining with Sirius Red of lung sections from control (PBS) or SARS-CoV-2 infected male (F) and female (M) golden hamsters, treated either with placebo or letrozole, at 21d p.i. Scale bar, $100 \mu \mathrm{m}(\mathrm{n}=5-7$ per group). $(\mathrm{G}, \mathrm{N})$ Weight loss of control (PBS) or SARS-CoV-2 infected male (G) and female (N) golden hamsters, treated either with placebo or letrozole, over a time course of 21 days $(n=5-7$ per group). Values are shown as means; error bars are shown as SD. Statistical significance was assessed by Mann-Whitney test or one-way ANOVA ( $\left.{ }^{\star *} p<0.01, * \star \star p<<0.001\right)$.

\section{Figure 5}

Genome-wide transcriptome analysis in the lungs of SARS-CoV-2 infected hamsters treated with the CYP19A1 inhibitor letrozole. The lung expression profiles of male and female golden hamsters, either non infected controls (PBS) or infected with SARS-CoV-2- or treated with placebo or with the CYP19A1 inhibitor letrozole were compared and are shown as ratio/intensity scatter plots (M/A-plot [M-value $=\log 2$ fold change; A-value = log2 base mean]). (A) Lung transcriptomes of SARS-CoV-2 infected and placebo treated male golden hamsters compared to control (PBS) and placebo treated male golden hamsters. (B) Lung transcriptomes of SARS-CoV-2 infected and placebo treated female golden hamsters compared to control (PBS) and placebo treated female hamsters. (C) Comparison of lung transcriptomes of SARSCoV-2 infected male golden hamsters, treated either with letrozole or placebo. (D) Lung transcriptomes of SARS-CoV-2 infected and letrozole treated female golden hamsters compared to SARS-CoV-2 infected and placebo treated female golden hamsters. (E) Heatmap depicting normalized expression of differentially expressed genes in the lung of male and female golden hamsters $21 \mathrm{~d}$ p.i., clustered according to their expression profiles (cut-off log 2 fold change $\geq 1$ or $\leq-1$, with an adjusted $p$-value $\leq 0.05$ ). (F-G) Significant differentially expressed gene in the lung of control (PBS) or SARS-CoV-2 infected male $(F)$ and female $(G)$ golden hamsters, treated either with placebo or letrozole. $(H)$ GO-term enrichment (biological process) of genes that were significantly downregulated in letrozole treated SARS-CoV-2 infected male golden hamsters compared to the placebo control group.

\section{Figure 6}

Association analysis of CYP19A1 gene variation in whole exome sequencing data of the GEN-COVID cohort. (A) Characteristics and COVID-19 severity grading of the GEN-COVID cohort used to understand if variants of the CYP19A1 gene were associated with COVID-19 severity in humans. (B) Ordered Logistic Regression (OLR) model in males, fitted using age to predict the ordinal grading $(0,1,2,3,4,5)$ dependent variable. On the $Y$ axis, the grading according to patients' treatment is reported. On the $X$ axis, age is reported. Red dots represent subjects falling above the expected treatment outcomes according to age (hence considered severe), blue dots are subjects falling below the expected treatment outcomes according to age (hence considered mild) and black dots are subjects matching the expected treatment outcomes according to age (hence considered intermediate. (C) Pie chart representing the number of 
male patients falling into "milder", "more severe" and "predicted by sex and age" categories. (D) Ordered Logistic Regression (OLR) model in females, as described in (B). (E) Pie chart representing the number of female patients falling into "milder", "more severe" and "predicted by sex and age" categories. (F) Association analysis between Thr201Met CYP19A1 and COVID-19 severity was performed by Chi-Square Test separately for males and females patients. A significant association was found in males cohort. (G) Forest plot summarizing the association results in males and females.

\section{Figure 7}

CYP19A1 expression in the lungs of fatal COVID-19 cases. (A) Detection of CYP19A1 protein (immunohistochemistry) and SARS-CoV-2 RNA (in situ hybridization) in the lungs of deceased male and female patients with COVID-19 or non-COVID-19 controls (representative pictures are shown) from the University Hospital Tübingen. The consecutive tissue sections reveal that CYP19A1 positive cells, most likely macrophages, are infected with SARS-CoV-2. Scale bar, $100 \mu \mathrm{m}$. (B) Detection of CYP19A1 and SARS-CoV-2 NP protein expression by immunohistochemistry of lungs from deceased male and female COVID-19 patients or non-COVID-19 controls (representative pictures are shown) from the University Hospital Hamburg-Eppendorf. Scale bar, $100 \mu \mathrm{m}$. (C) Detection of CYP19A1 protein expression by immunohistochemistry of lungs from deceased male COVID-19 patients or non-COVID-19 controls (representative pictures are shown) from the Erasmus Medical Center Rotterdam. Scale bar, $50 \mu \mathrm{m}$. (D) CYP19A1 mRNA expression levels in the lungs from deceased male COVID-19 patients and non-COVID-19 controls at the University Hospital Tübingen (non-COVID-19: $n=8$, COVID-19: $n=9$ ). (E) CYP19A1 mRNA expression levels in the lungs from deceased male COVID-19 patients and non-COVID-19 controls at the University Hospital Hamburg-Eppendorf (non-COVID-19: $n=3$, COVID-19: $n=12$ ). (E) CYP19A1 mRNA expression levels in the lungs from deceased male COVID-19 patients and non-COVID-19 controls at the Erasmus Medical Center Rotterdam (non-COVID-19: $n=6$, COVID-19: $n=11$ ). Relative CYP19A1 mRNA expression values in non-COVID-19 controls were set to 1 after normalization against RPL32 (D-F). Values are shown as means; error bars are shown as SD. Statistical significance was assessed by Mann-Whitney test $(\star \star p<0.01, \star \star \star p<0.001)$.

\section{Supplementary Files}

This is a list of supplementary files associated with this preprint. Click to download.

- FigureS1.png

- FigureS2.png

- FigureS3.png

- FigureS4.png

- FigureS5.png

- FigureS6.png 
- FigureS7.png

- FigureS8.png

- FigureS9.png

- FigureS10.png

- Figures11.png

- FigureS12.png

- FigureS13.png

- TableS2.xIsx

- TableS1.xlsx 